\title{
Entanglement of a System of an Indirectly Linked Two-Coupled-Cavity through an Optical Fiber Cable for Single Excitation Atomic States in the Presence of an External Field
}

Sadek Ahmed Hanoura ( $\nabla$ sadek.ahmed10@yahoo.com )

Sinai University - El Arish Campus https://orcid.org/0000-0001-6283-4500

\section{Research Article}

Keywords: External classical field, Entanglement, von Neumann atomic entropy, Optical fiber cable modes, Effective Hamiltonians, Time average theory.

Posted Date: August 13th, 2021

DOI: https://doi.org/10.21203/rs.3.rs-583737/v1

License: (c) (1) This work is licensed under a Creative Commons Attribution 4.0 International License. Read Full License 


\title{
Entanglement of a System of an Indirectly Linked Two-Coupled-Cavity through an Optical Fiber Cable for Single Excitation Atomic States in the Presence of an External Field
}

\author{
S. A. Hanoura ${ }^{1 *}$, M. M. A. Ahmed ${ }^{2}$, E. M. Khalil ${ }^{2,3}$, and A.-S. F. Obada ${ }^{2}$ \\ 1-Department of Basic Science, Faculty of Engineering, Sinai University, Al-Arish, Egypt \\ 2-Department of Mathematics, Faculty of Science, Al-Azhar \\ University, Nasr City, 11884, Cairo Egypt. \\ 3-Department of Mathematics, Faculty of Science, Taif University, Taif, Saudi Arabia.
}

\begin{abstract}
The dynamics of the quantum entropies of a system of two cavities coupled by an optical fiber cable, each cavity contains a two-level atom interacting with a single electromagnetic field in addition to an external classical field, is investigated. Under canonical transformations, the considered Hamiltonian is diagonalized. Effective Hamiltonians in three different limiting regimes: namely large optical fiber cable coupling bstrength, large detunig, and comparable detuning and optical fiber cable coupling strength, are derived. The $i^{\text {th }}$-tom are respectively prepared in the superposition coherent and the ground states while the fields are prepared in the vacuum states. An analytical expression for the solution of the Schrödinger equation for each dispersive is derived. The degree of entanglement (DEM) is studied by using von Neumann atomic entropies. The influences of both the optical fiber cable coupling strength and the detuning on the evolution of the DEM "their values are closely chosen to be compatible with the imposed restrictions for the applications of the different regimes" are analyzed. General conclusions reached are illustrated by numerical results.
\end{abstract}

Key words: External classical field; Entanglement; von Neumann atomic entropy; Optical fiber cable modes; Effective Hamiltonians; Time average theory.

The dynamics of the DEM functions and the excitations of the different modes is analyzed. The time average effective Hamiltonians are formulated in the framework of the James's theory. The effect of the system parameters is investigated in accordance with certain dispersive regimes. The rate of the energy transfer between the atomic subsystems is controlled by certain factor which is vary from one dispersive to another.

\section{Introduction}

Cavity quantum electrodynamic (QED) is one of the approaches for quantum computations. It is not confined to quantum computations but is also used for the quantum information [1] and for the quantum gate operations [2,3] and circuit QED [4]. Entanglement is a property found in the composite quantum systems, where the correlation between the subsystems can not be discussed classically.

Entanglement has been widely investigated in quantum information processing [5-9]. It plays important roles in many potential applications, such as quantum communication, quantum teleportation, quantum cryptography, entanglement swapping, dense coding, and quantum computing, etc [10-16]. The degree of entanglement (DEM) can be studied via different measures such as von Neumann entropy, linear entropy, negativity and others measurements.

Quantifying the DEM requires knowledge of the state of the system. If the state of the system is a pure, then it is sufficient to use the von Neumann entropy $[17,18]$. The time evolution of atomic (field) entropy reflects the time evolution of the degree of entanglement thus when the entropy is high, the degree of entanglement is strong.

*e-mail: sadek.ahmed10@yahoo.com 
A model which describes the interaction of a quantized field and a two-level atom within the rotating wave approximation is dubbed Jaynes-Cummings model (JCM) [19]. Many researchers turned their attention to extend and generalize this model. In recent years much attention has been focused on the properties of the entanglement between the field and the atom. In particular, the entropy of a two-level system interacting with a single-mode [20-28], and two-mode $[29,30]$ has been investigated. Also, atom-field entanglement in multi-photon processes for a single atom either with a one-mode or with a two-mode field [21,31-34], and a two-atom system has been studied [20,27,35-38]. A tentative approach to entanglement measures for a system of a three-level atom interacting with a quantized cavityfield has been introduced [39]. Furthermore, the evolution of the (atomic) field entropy for the three-level atom one-mode [40-51] and two-mode [52-55] model has been studied. On the other hand, the system of a four-level atom interacting with a single mode of electromagnetic field has been investigated [56-61].

The coupling of the distant qubits is one requirement of distributed quantum information processing to produce state transfer. This is the way to the entanglement generation or quantum gate operations between separate nodes of the systems. The strong coupling that can be achieved between atoms and cavity modes can be used to perform logic operations. Atoms are qualified to storing qubits in their internal states for long-lived internal states, while, the photons are the best carriers for information over long distances. Also, the cavities can provide a good insulation against the environment.

Nowadays, the researches turn attentions to engineer the quantum states transfer via the photonic processes. Then, they postulated several techniques to control the coupling between two successive nodes. One of these techniques is to connect the successive cavities indirectly by a quantum channel such as optical fiber cables [62,63]. Another common technique is to connect the successive cavities directly by the overlap of evanescent cavity fields which permits photons to hop from one cavity to the other and vice versa [64-67]. The model which describes the physics of interacting bosons on a lattice is called the Bose-Hubbard model. The model was first introduced by Gersch and Knollman in 1963. The time evolution of remote cavities has been investigated [68-79]. The cavity field modes are initially either in vacuum state or in a correlated state [80-84].

Nowadays, there are good growing interests in the control and manipulation of quantum light because of its applications in quantum information processing. Controlling and manipulation of the quantum light have been performed by many theories. The latest and the most common is that introduced by Daniel F. V. James [85]. Based on such a method, not only quantum correlations can be shaped, but also the initial states can be easily identified. Coupled cavities models have been studied mathematically in detail with the aid of theory. The authors in [86] treated the problem of two coupled cavities through theory introduced by James [85].

To couple the driving field with the JCM there are usually two different ways, either to couple the driving field to the atom or to use an external field to drive the cavity mode [87]. The spectroscopy of the Jayens-Cummings "molecule" is done via an extra field. Where it is used in the detection of the Fock-state of the radiation field and in a quantum phase gate, in addition to the creation of multi-particle entangled states [88-91]. Actually, this encouraged the researchers to pay an attention to examine the effect of the external field on this model, through variety of various techniques.

This paper is organized as follows: In Section-2, we introduce the Hamiltonian model through the local modes, rephrasing it in terms of the delocalized modes, and the effective Hamiltonians for three dispersive regimes using a method introduced in Ref. [85]. In Section-3, we derive analytical expressions for the coefficients of the wave functions of the Hamiltonians via the solution of the Schrödinger equation. In Section-4, we employ the analytical results in Section-3 to investigate the properties of the DEM through the von Neumann entropy. We devote Section$\mathbf{5}$ to discuss the resulting features and phenomena due to von Neumann entropy. Finally, we give our conclusion in Section-6.

\section{The Model}

The considered model is a coupled two Jaynes-Cummings model cavities; each of which contains a single two-level atom interacts separately with a quantized cavity field and they are coupled through an optical fiber mode, as shown in Fig.(1). Moreover, each atom is driven by on resonance an external classical field. The total Hamiltonian of the considered system can be cast as follows (we adopt $\hbar=1$ ):

$$
\hat{H}=\sum_{j=1}^{2}\left[\omega_{j} \hat{a}_{j}^{+} \hat{a}_{j}+\frac{\Omega_{j}}{2} \sigma_{j}^{z}\right]+\omega_{f} \hat{b}^{+} \hat{b}+\sum_{j=1}^{2} i \lambda_{j}\left(\hat{a}_{j}+\hat{a}_{j}^{+}\right)\left(\hat{\sigma}_{j}^{+}-\hat{\sigma}_{j}^{-}\right)+\left[\sum_{j=1}^{2} g_{j} \hat{\sigma}_{j}^{+}+\nu b\left(\hat{a}_{1}^{+}+\hat{a}_{2}^{+}\right)+H . C .\right] .
$$


The $\omega_{j}, \omega_{f}$, and $\Omega_{j}$ represent the frequencies of the quantized fields, the frequency of the optical fiber mode, and the frequencies of the atomic transitions respectively. Moreover, the $\lambda_{j}, g_{j}$, and $\nu$ refer to the atom-field coupling in the $j^{\text {th }}$ cavity, the external classical field coupling, and the strength of the optical fiber cable coupling respectively. The operators $\hat{a}_{j}^{+}$, and $\hat{a}_{j}$ are boson operators of the fields which obey the commutation relations $\left[\hat{a}_{j}, \hat{a}_{k}^{+}\right]=\hat{I} \delta_{j k}$. The boson operators $\hat{b}^{+}$, and $\hat{b}$ represent the fields mode which obey $\left[\hat{b}, \hat{b}^{+}\right]=\hat{I}$. The operators $\hat{\sigma}_{j}^{-}, \hat{\sigma}_{j}^{+}$, and $\sigma_{j}^{z}$ are Pauli operators defined by $|g\rangle_{j}\langle e|,| e\rangle_{j}\langle g|$, and $|e\rangle_{j}\langle e|-| g\rangle_{j}\langle g|$ respectively. The kets $|e\rangle_{j}$, and $|g\rangle_{j}$ represent the excited and ground states of the $j^{\text {th }}$-atom respectively. The operators $\hat{\sigma}_{j}^{-}, \hat{\sigma}_{j}^{+}$, and $\sigma_{j}^{z}$ satisfy $\left[\sigma_{j}^{z}, \hat{\sigma}_{j}^{ \pm}\right]= \pm 2 \hat{\sigma}_{j}^{ \pm} \delta_{i j}$, and $\left[\hat{\sigma}_{j}^{+}, \hat{\sigma}_{j}^{-}\right]=\sigma_{j}^{z} \delta_{i j}$.

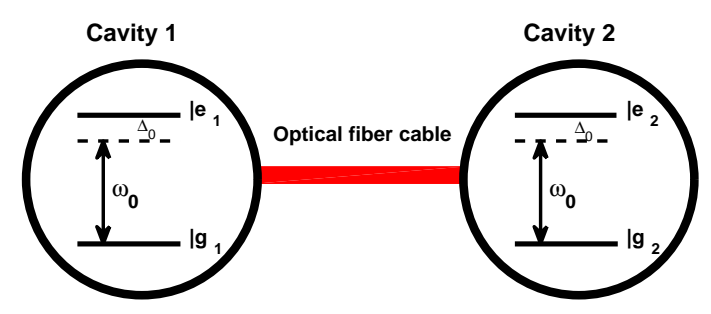

\subsection{Canonical Transformation}

The Hamiltonian in Eq.(1) as it stands is very hard to tackle. Thus, in what follow, we remove the classical field whereupon remove the converter part through two well-known canonical transformations [79, 86, 92-96].

\subsubsection{Eliminating the Classical Field}

To remover the external classical field term, we apply the following transformation:-

$$
\left(\begin{array}{c}
\hat{\sigma}_{j}^{z} \\
\hat{\sigma}_{j}^{+} \\
\hat{\sigma}_{j}^{-}
\end{array}\right)=\left(\begin{array}{ccc}
\cos \left(2 \zeta_{j}\right) & -\sin \left(2 \zeta_{j}\right) & -\sin \left(2 \zeta_{j}\right) \\
\frac{1}{2} \sin \left(2 \zeta_{j}\right) & \cos ^{2} \zeta_{j} & -\sin ^{2} \zeta_{j} \\
\frac{1}{2} \sin \left(2 \zeta_{j}\right) & -\sin ^{2} \zeta_{j} & \cos ^{2} \zeta_{j}
\end{array}\right)\left(\begin{array}{c}
\hat{S}_{j}^{z} \\
\hat{S}_{j}^{+} \\
\hat{S}_{j}^{-}
\end{array}\right)
$$

Consequently,

$$
\hat{\sigma}_{j}^{+}-\hat{\sigma}_{j}^{-}=S_{j}^{+}-S_{j}^{-}
$$

When we choose $\zeta_{j}$ to be: $\zeta_{j}=\frac{1}{2} \arccos \left(\frac{\Omega_{j}}{\sqrt{\Omega_{j}^{2}+4 g_{j}^{2}}}\right)$, and apply the rotating wave approximation, the Hamiltonian in Eq.(1) is reduced to

$$
\hat{\mathrm{H}}=\sum_{j=1}^{2}\left[\omega_{j} \hat{a}_{j}^{+} \hat{a}_{j}+\frac{\mho_{j}}{2} S_{j}^{z}+i \lambda_{j}\left(\hat{a}_{j} \hat{S}_{j}^{+}-\hat{a}_{j}^{+} \hat{S}_{j}^{-}\right)\right]+\omega_{f} \hat{b}^{+} \hat{b}+\nu\left(\hat{b}\left(\hat{a}_{1}^{+}+\hat{a}_{2}^{+}\right)+\hat{b}^{+}\left(\hat{a}_{1}+\hat{a}_{2}\right)\right) .
$$

The new states consistent with the rotated operators are given by:-

$$
\begin{aligned}
|+\rangle_{j} & =\cos \zeta_{j}|e\rangle_{j}+\sin \zeta_{j}|g\rangle_{j} \\
|-\rangle_{j} & =\cos \zeta_{j}|g\rangle_{j}-\sin \zeta_{j}|e\rangle_{j}
\end{aligned}
$$

It is obvious that this transformation results not only in removing the external classical field, but also causes an augmentation of the atomic frequency $\Omega_{j}$ to the shifted atomic frequency $\mho_{j}=\sqrt{\Omega_{j}^{2}+4 g_{j}^{2}}$. Further, the new atomic operators become $\hat{S}_{j}^{ \pm}=| \pm\rangle_{j}\langle\mp|$, and $\hat{S}_{j}^{z}=|+\rangle_{j}\langle+|-|-\rangle_{j}\langle-|$ satisfying the same commutation relations as $\hat{\sigma}_{j}^{ \pm}$, and $\hat{\sigma}_{j}^{z}$.

\subsubsection{Eliminating the Converter Part}

The elimination of the converter part can be made via an unitary canonical transformation; in it, the delocalized operators are controlled by:- $\hat{b}=\frac{\hat{C}_{1}-\hat{C}_{2}}{\sqrt{2}}, \hat{a}_{1}=\frac{1}{2}\left(\hat{C}_{1}+\hat{C}_{2}+\sqrt{2} \hat{C}_{0}\right), \hat{a}_{2}=\frac{1}{2}\left(\hat{C}_{1}+\hat{C}_{2}-\sqrt{2} \hat{C}_{0}\right)$. The operators $C_{i}$ satisfy the boson commutation relations $\left[\hat{C}_{i}, \hat{C}_{j}^{+}\right]=\hat{I} \delta_{i j}$. Thus, the Hamiltonian in Eq.(4) takes the form:

$$
\hat{H}=\hat{H}_{0}+\hat{H}_{i n t} .
$$


$\hat{H}_{0}$ : is the free atomic and field Hamiltonian while $\hat{H}_{\text {int }}$ : is the interaction Hamiltonian, and all formed as follows:-

$$
\begin{aligned}
\hat{H}_{0} & =\sum_{\ell=0}^{2} \tilde{\omega}_{\ell} \hat{C}_{\ell}^{+} \hat{C}_{\ell}+\sum_{j=1}^{2}\left[\frac{\mho_{j}}{2} \hat{S}_{j}^{z}\right] \\
\hat{H}_{i n t} & =i \sum_{j=1}^{2} \frac{\lambda_{j}}{2}\left[\left(\hat{C}_{1}+\hat{C}_{2}-(-)^{j} \sqrt{2} \hat{C}_{0}\right) \hat{S}_{j}^{+}-H . C .\right]
\end{aligned}
$$

with $\tilde{\omega}_{\ell}=\omega_{0}-(-)^{\ell} \sqrt{2} \nu\left(\delta_{1 \ell}+\delta_{2 \ell}\right)$. The immediately depicted relations are formed while the resonance of the bosonic subsystems is taken into account, namely $\omega_{j}=\omega_{0}=\omega_{f}$.

\subsection{The Interaction Picture}

The interaction picture of the Hamiltonian in Eq.(6), is given by

$$
\hat{H}_{I}=\exp \left(i t \hat{H}_{0}\right) \hat{H}_{i n t} \exp \left(-i t \hat{H}_{0}\right)
$$

By using the Baker-Campbell-Hausdorff formula [97], we obtain:

$$
\hat{H}_{I}=\hat{H}_{i n t}+(i t)\left[\hat{H}_{0}, \hat{H}_{i n t}\right]+\frac{(i t)^{2}}{2 !}\left[\hat{H}_{0},\left[\hat{H}_{0}, \hat{H}_{i n t}\right]\right]+\frac{(i t)^{3}}{3 !}\left[\hat{H}_{0},\left[\hat{H}_{0},\left[\hat{H}_{0}, \hat{H}_{i n t}\right]\right]\right]+\ldots .
$$

Thus,

$$
\hat{H}_{I}=\sum_{\sigma=1}^{2} \frac{i \lambda_{\sigma}}{2}\left[\left(\sum_{j=1}^{2} \hat{C}_{j} e^{-i t\left(\tilde{\omega}_{j}-\mho_{\sigma}\right)}+\sqrt{2} \hat{C}_{0} e^{-i t\left(\omega_{0}-\mho_{\sigma}\right)}\right) \hat{S}_{\sigma}^{+}-H . C .\right] .
$$

This interaction picture plays a crucial role in calculating many quantum aspects formulae and their investigation. In what follow, we shall show its action in derivation of the effective Hamiltonian according to several dispersive regimes.

\subsection{The Effective Hamiltonian}

The effective Hamiltonian $\left(s a y, \hat{H}_{e f f}\right)$ can be obtained with several techniques. One of them is introduced in [85]. It is defined by the following relation

$$
\hat{H}_{e f f}=\overline{\hat{H}_{I}(t)}+\frac{1}{2}\left(\overline{\left[\hat{H}_{I}(t), \hat{V}_{1}(t)\right]}-\left[\overline{\hat{H}_{I}(t)}, \overline{\hat{V}_{1}(t)}\right]\right), \quad \hat{V}_{1}(t)=-i \int_{t_{0}}^{t} \hat{H}_{I}\left(t^{\prime}\right) d t^{\prime}
$$

Thus

$$
\hat{V}_{1}(t)=\sum_{\sigma=1}^{2} \frac{i \lambda_{\sigma}}{2}\left[\left(\sum_{j=1}^{2} \frac{\hat{C}_{j}}{\tilde{\omega}_{j}-\mho_{\sigma}} e^{-i t\left(\tilde{\omega}_{j}-\mho_{\sigma}\right)}+\sqrt{2} \frac{\hat{C}_{0}}{\omega_{0}-\mho_{\sigma}} e^{-i t\left(\omega_{0}-\mho_{\sigma}\right)}\right) \hat{S}_{\sigma}^{+}+H . C .\right]
$$

Now, we are proceeding to obtain the effective Hamiltonians in three dispersive regimes approximations, they are, (i) Large cavity-fiber-cavity coupling strength (ii) Large detuning, and (iii) Comparable cavity-fiber-cavity coupling strength and detuning. To make the calculations analytically easier, we assume symmetry of the atomic subsystems (i.e., $\Omega_{j}=\Omega$ ) besides the symmetric couplings case $\left(i . e ., \lambda_{j}=\lambda\right.$ ). The detuning between the atomic modes $S_{j}$, and $C_{j}$ are $\Delta_{\iota}=\mho_{1}-\omega_{\iota}, \iota=0,1,2$.

\subsubsection{Large Optical Fiber Cable Strength}

This approximation is applicable when the condition $\sqrt{2} \nu>\Delta_{0}, \lambda$ is satisfied. Moreover, we assume that $\Delta_{0}, \Delta_{1}$, and $\Delta_{2}>\lambda$. These conditions ensure vanishing of the time average of both $\hat{V}_{1}(t)$ and $\hat{H}_{I}(t)$. The effective Hamiltonian for this regime can thus be written in the form:

$$
\begin{aligned}
\hat{H}_{e f f} & =\hat{H}_{0}+\frac{\lambda^{2}}{4}\left[\frac{\hat{C}_{1} \hat{C}_{1}^{+}}{\Delta_{1}}+\frac{\hat{C}_{2} \hat{C}_{2}^{+}}{\Delta_{2}}+\frac{2 \hat{C}_{0} \hat{C}_{0}^{+}}{\Delta_{0}}\right]\left(|+\rangle_{1}\langle+|+|+\rangle_{2}\langle+|\right) \\
& -\frac{\lambda^{2}}{4}\left[\frac{\hat{C}_{1}^{+} \hat{C}_{1}}{\Delta_{1}}+\frac{\hat{C}_{2}^{+} \hat{C}_{2}}{\Delta_{2}}+\frac{2 \hat{C}_{0}^{+} \hat{C}_{0}}{\Delta_{0}}\right]\left(|-\rangle_{1}\langle-|+|-\rangle_{2}\langle-|\right) \\
& +\frac{\lambda^{2}}{4}\left[\frac{1}{\Delta_{1}}+\frac{1}{\Delta_{2}}-\frac{2}{\Delta_{0}}\right]\left(\hat{S}_{2}^{-} \hat{S}_{1}^{+}+\hat{S}_{1}^{-} \hat{S}_{2}^{+}\right)
\end{aligned}
$$




\subsubsection{Large Detuning}

According to this approximation, the condition that $\Delta_{0}>\sqrt{2} \nu, \lambda$ has to be satisfied. Moreover, we assume that $\Delta_{1}, \Delta_{2}>\lambda$. This condition ensures vanishing of the time average of both $\hat{V}_{1}(t)$ and $\hat{H}_{I}(t)$. Thus, the effective Hamiltonian obtained in this case, is written as:

$$
\begin{aligned}
\hat{H}_{e f f} & =\hat{H}_{0}+\frac{\lambda^{2}}{8}\left[\frac{2 \hat{C}_{1} \hat{C}_{1}^{+}}{\Delta_{1}}+\frac{2 \hat{C}_{2} \hat{C}_{2}^{+}}{\Delta_{2}}+\frac{4 \hat{C}_{0} \hat{C}_{0}^{+}}{\Delta_{0}}\right]\left(\hat{S}_{1}^{z}+\hat{S}_{2}^{z}\right) \\
& +\frac{\lambda^{2}}{4}\left[\frac{1}{\Delta_{1}}+\frac{1}{\Delta_{2}}+\frac{2}{\Delta_{0}}\right]\left(\hat{S}_{1}^{-} \hat{S}_{1}^{+}+\hat{S}_{2}^{-} \hat{S}_{2}^{+}\right) \\
& +\frac{\lambda^{2}}{4}\left[\frac{1}{\Delta_{1}}+\frac{1}{\Delta_{2}}-\frac{2}{\Delta_{0}}\right]\left(\hat{S}_{2}^{-} \hat{S}_{1}^{+}+\hat{S}_{1}^{-} \hat{S}_{2}^{+}\right) \\
& +\frac{\lambda^{2}}{8}\left(\frac{1}{\Delta_{1}}+\frac{1}{\Delta_{2}}\right)\left[\hat{C}_{1} \hat{C}_{2}^{+}+\hat{C}_{1} \hat{C}_{2}^{+}\right]\left(\hat{S}_{1}^{z}+\hat{S}_{2}^{z}\right) \\
& +\frac{\lambda^{2} \sqrt{2}}{8}\left[\sum_{j=1}^{2} \hat{C}_{j} \hat{C}_{0}^{+}\left(\frac{1}{\Delta_{0}}+\frac{1}{\Delta_{j}}\right)+h . c .\right]\left(\hat{S}_{1}^{z}-\hat{S}_{2}^{z}\right) .
\end{aligned}
$$

\subsubsection{Comparable Optical Fiber Cable Coupling Strength and Detuning}

For the present approximation, the transition frequency of one of the atoms is assumed to be nearly resonant with one of the delocalized photonic modes. Such an assumption can be written as $\Delta_{1} \approx 0$. Applying the condition and taking the time average, thus, the effective Hamiltonian is written as:-

$$
\begin{aligned}
\hat{H}_{e f f} & =\hat{H}_{0}+\frac{i \lambda}{2}\left[\hat{C}_{1}\left(\hat{S}_{1}^{+}+\hat{S}_{2}^{+}\right)-\hat{C}_{1}^{+}\left(\hat{S}_{1}^{-}+\hat{S}_{2}^{-}\right)\right]+\frac{\lambda^{2}}{4}\left[\frac{\hat{C}_{2} \hat{C}_{2}^{+}}{\Delta_{2}}+\frac{2 \hat{C}_{0} \hat{C}_{0}^{+}}{\Delta_{0}}\right] \sum_{j=1}^{2}|+\rangle_{j}\langle+| \\
& -\frac{\lambda^{2}}{4}\left[\frac{\hat{C}_{2}^{+} \hat{C}_{2}}{\Delta_{2}}+\frac{2 \hat{C}_{0}^{+} \hat{C}_{0}}{\Delta_{0}}\right] \sum_{j=1}^{2}|-\rangle_{j}\langle-|+\frac{\lambda^{2}}{4}\left[\frac{1}{\Delta_{2}}-\frac{2}{\Delta_{0}}\right]\left(\hat{S}_{2}^{-} \hat{S}_{1}^{+}+\hat{S}_{1}^{-} \hat{S}_{2}^{+}\right) .
\end{aligned}
$$

To make calculations easier, we concern obtaining the wave functions of the different Hamiltonians in absence of the external classical field.

\section{The Wave Function}

Based on what we have mentioned, the initial state of the considered system in the localized basis is

$$
\left|\Psi_{A F}(0)\right\rangle_{o l d}=\cos \theta|e g 000\rangle_{o l d}+\sin \theta|g g 000\rangle_{o l d}
$$

Knowing that $|000\rangle_{\text {new }}=|000\rangle_{\text {old }}$. Consequently, the final state of the considered system in the framework of the delocalized basis can be formulated as:

$$
\left|\Psi_{A F}(t)\right\rangle_{n e w}=\sum_{j=1}^{6} A_{j}(t)\left|\psi_{j}\right\rangle_{n e w}
$$

Now, the main target is to solve the Schrödinger equation $\left(i . e ., \hat{H}\left|\Psi_{A F}(t)\right\rangle=i\left|\dot{\Psi}_{A F}(t)\right\rangle\right)$ for the full Hamiltonian and for the three effective Hamiltonians resulted in three different regimes mentioned before. The wave function is assumed initially normalized then it stays normalized (namely, $\sum_{j=1}^{6}\left|A_{j}(t)\right|^{2}=1$ ) as it evolves in time. The coefficients of the wave function in the framework of the localized basis are from their counterparts in the framework of the delocalized basis obtained after using

$$
\begin{aligned}
|100\rangle_{\text {new }} & =\frac{1}{2}(|100\rangle+|010\rangle+\sqrt{2}|001\rangle)_{\text {old }} \\
|010\rangle_{\text {new }} & =\frac{1}{2}(|100\rangle+|010\rangle-\sqrt{2}|001\rangle)_{\text {old }} \\
|001\rangle_{\text {new }} & =\frac{1}{\sqrt{2}}(|100\rangle-|010\rangle)_{\text {old }} .
\end{aligned}
$$


Then

$$
\left|\Psi_{A F}(t)\right\rangle_{o l d}=\sum_{j=1}^{6} B_{j}(t)\left|\psi_{j}\right\rangle_{o l d}
$$

with $B_{\ell}(t)=A_{\ell}(t), B_{4,5}(t)=\left(\frac{A_{4}(t)}{2}+\frac{A_{5}(t)}{2} \pm \frac{A_{6}(t)}{\sqrt{2}}\right), B_{6}(t)=\frac{1}{\sqrt{2}}\left(A_{4}(t)-A_{5}(t)\right), \ell=1,2,3$. Moreover, $\left|\psi_{1}\right\rangle_{m}=|e g 000\rangle$, $\left|\psi_{2}\right\rangle_{m}=|g e 000\rangle,\left|\psi_{3}\right\rangle_{m}=|g g 000\rangle,\left|\psi_{4}\right\rangle_{m}=|g g 100\rangle,\left|\psi_{5}\right\rangle_{m}=|g g 010\rangle$, and $\left|\psi_{6}\right\rangle_{m}=|g g 001\rangle$, with $m=$ old, new. In what follows, we present the solution for the wave functions for the different Hamiltonians.

\subsection{The Full Hamiltonian}

For the Hamiltonian in Eq.(6), the coefficients of the final state are given by:-

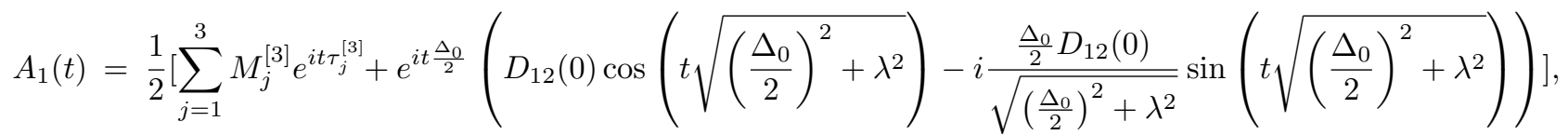

$$
\begin{aligned}
& A_{2}(t)=\frac{1}{2}\left[\sum_{j=1}^{3} M_{j}^{[3]} e^{i t \tau_{j}^{[3]}}-e^{i t \frac{\Delta_{0}}{2}}\left(D_{12}(0) \cos \left(t \sqrt{\left(\frac{\Delta_{0}}{2}\right)^{2}+\lambda^{2}}\right)-i \frac{\frac{\Delta_{0}}{2} D_{12}(0)}{\sqrt{\left(\frac{\Delta_{0}}{2}\right)^{2}+\lambda^{2}}} \sin \left(t \sqrt{\left(\frac{\Delta_{0}}{2}\right)^{2}+\lambda^{2}}\right)\right]\right. \\
& A_{3}(t)=A_{3}(0) e^{i t \mho_{1}}, \quad A_{4}(t)=\sum_{j=1}^{3} M_{j}^{[1]} e^{i t\left(\tau_{j}^{[1]}+\Delta_{1}\right)}, \quad A_{5}(t)=\sum_{j=1}^{3} M_{j}^{[2]} e^{i t\left(\tau_{j}^{[2]}+\Delta_{2}\right)}, \\
& A_{6}(t)=e^{i t \frac{\Delta_{0}}{2}} \frac{-\frac{\lambda}{\sqrt{2}} D_{12}(0)}{\sqrt{\left(\frac{\Delta_{0}}{2}\right)^{2}+\lambda^{2}}} \sin \left(t \sqrt{\left(\frac{\Delta_{0}}{2}\right)^{2}+\lambda^{2}}\right), \quad D_{12}(0)=A_{1}(0)-A_{2}(0)
\end{aligned}
$$

The eigenvalues $\tau_{j}^{[\ell]}(\ell, j=1,2,3)$ are controlled by:-

$$
\tau_{j}^{[\ell]}=-\frac{X_{1}}{3}+\frac{2}{3}\left(\sqrt{X_{1}^{2}-3 X_{2}}\right) \cos \left(\theta_{j}^{[\ell]}\right), j, \ell=1,2,3,
$$

where, the parameters $\theta_{j}^{[\ell]}(\ell, j=1,2,3)$ are given by:-

$$
\theta_{j}^{[\ell]}=\left(\frac{1}{3} \cos ^{-1}\left[\frac{9 X_{1} X_{2}-2 X_{1}^{3}-27 X_{3}}{2\left(X_{1}^{2}-3 X_{2}\right)^{\frac{3}{2}}}\right]+(j-1) \frac{2 \pi}{3}\right) .
$$

For $A_{4}(t)$, the coefficients $X_{1}, X_{2}$, and $X_{3}$ are given by:-

$$
\begin{aligned}
& X_{1}=2 \Delta_{1}-\Delta_{2}, \\
& X_{2}=-\left(\lambda^{2}+\Delta_{1} \Delta_{2}-\Delta_{1}^{2}\right), \\
& X_{3}=\frac{\lambda^{2}}{2}\left(\Delta_{2}-\Delta_{1}\right) .
\end{aligned}
$$

For $A_{5}(t)$, the coefficients $X_{1}, X_{2}$, and $X_{3}$ are given by:-

$$
\begin{aligned}
& X_{1}=2 \Delta_{2}-\Delta_{1} \\
& X_{2}=-\left(\lambda^{2}+\Delta_{2} \Delta_{1}-\Delta_{2}^{2}\right), \\
& X_{3}=\frac{\lambda^{2}}{2}\left(\Delta_{1}-\Delta_{2}\right) .
\end{aligned}
$$

For $A_{1,2}(t)$, the coefficients $X_{1}, X_{2}$, and $X_{3}$ are given by:-

$$
\begin{aligned}
& X_{1}=-\left(\Delta_{1}+\Delta_{2}\right), \\
& X_{2}=-\left(\lambda^{2}-\Delta_{1} \Delta_{2}\right), \\
& X_{3}=\frac{\lambda^{2}}{2}\left(\Delta_{1}+\Delta_{2}\right) .
\end{aligned}
$$

Meanwhile, the coefficients $M_{1}^{[j]}, M_{2}^{[j]}$, and $M_{3}^{[j]}$ are controlled by:-

$$
\left(\begin{array}{c}
M_{1}^{[j]} \\
M_{2}^{[j]} \\
M_{3}^{[j]}
\end{array}\right)=\left(\begin{array}{ccc}
1 & 1 & 1 \\
\tau_{1}^{[\mathrm{j}]} \mathrm{i} & \tau_{2}^{[\mathrm{j}]} \mathrm{i} & \tau_{3}^{[\mathrm{j}]} \mathrm{i} \\
-\left(\tau_{1}^{[\mathrm{j}]}\right)^{2} & -\left(\tau_{2}^{[\mathrm{j}]}\right)^{2} & -\left(\tau_{3}^{[\mathrm{j}]}\right)^{2}
\end{array}\right)^{-1}\left(\begin{array}{c}
\mu_{j}^{(1)}(0) \\
\mu_{j}^{(2)}(0) \\
\mu_{j}^{(3)}(0)
\end{array}\right)
$$


with $\left(\mu_{1}, \mu_{2}, \mu_{3}\right)=\left(\tilde{A}_{4}(t), \tilde{A}_{5}(t), \tilde{S}_{12}(t)\right)$, where $\tilde{A}_{k+3}^{(j-1)}(0)$, and $\tilde{S}_{12}^{(j-1)}(0)(k=1,2)$ are controlled by:-

$$
\begin{aligned}
& \tilde{A}_{k+3}^{(j-1)}(0)=-\left[\frac{\lambda}{2} \delta_{j 2}-i \frac{\Delta_{k} \lambda}{2} \delta_{j 3}\right]\left(A_{1}(0)+A_{2}(0)\right), \\
& \tilde{S}_{12}^{(j-1)}(0)=\left[\delta_{j 1}-\lambda^{2} \delta_{j 3}\right]\left(A_{1}(0)+A_{2}(0)\right) .
\end{aligned}
$$

Moreover, for $A(t)_{1,2,4,5}$, we have

$$
\theta_{j}^{[\ell]}=\left(\frac{1}{3} \cos ^{-1}\left[\frac{\frac{1}{2}\left(\Delta_{1}+\Delta_{2}\right)\left(4 \Delta_{1}^{2}-10 \Delta_{1} \Delta_{2}+4 \Delta_{2}^{2}-9 \lambda^{2}\right)}{2\left(\Delta_{1}^{2}-\Delta_{1} \Delta_{2}+\Delta_{2}^{2}+3 \lambda^{2}\right)^{\frac{3}{2}}}\right]+(j-1) \frac{2 \pi}{3}\right) .
$$

\subsection{Large Optical Fiber Cable Strength and Detuning}

For the Hamiltonians in Eqs. $(13,14)$, the non vanishing coefficients of the final state are given by:-

$$
\begin{aligned}
& A_{1}(t)=e^{-i t \kappa_{00}}\left[A_{1}(0) \cos \left(t \kappa_{11}\right)-i A_{2}(0) \sin \left(t \kappa_{11}\right)\right], \\
& A_{2}(t)=e^{-i t \kappa_{00}}\left[A_{2}(0) \cos \left(t \kappa_{11}\right)-i A_{1}(0) \sin \left(t \kappa_{11}\right)\right] \text {, } \\
& A_{3}(t)=A_{3}(0) e^{i t \mho_{1}} \text {, }
\end{aligned}
$$

where both $\kappa_{00}$, and $\kappa_{11}$ are controlled by: $\kappa_{00}=\frac{\lambda^{2}}{4}\left(\frac{1}{\Delta_{1}}+\frac{1}{\Delta_{2}}+\frac{2}{\Delta_{0}}\right)$, and $\kappa_{11}=\frac{\lambda^{2}}{4}\left(\frac{1}{\Delta_{1}}+\frac{1}{\Delta_{2}}-\frac{2}{\Delta_{0}}\right)$.

\subsection{Comparable Optical Fiber Cable Coupling Strength and Detuning}

For the Hamiltonian in Eq.(15), the non vanishing coefficients of the final state are obtained by:-

$$
\begin{aligned}
& A_{1}(t)=\frac{1}{2} e^{-i t \frac{\lambda^{2}}{2}\left(\frac{1}{\Delta_{2}}+\frac{2}{\Delta_{0}}\right)}\left(+D_{12}(0) e^{i t \frac{\lambda^{2}}{4}\left(\frac{1}{\Delta_{2}}-\frac{2}{\Delta_{0}}\right)}+e^{0.5 i t\left(\Delta_{1}+\frac{\lambda^{2}}{\Delta_{0}}\right)}\left[e_{1} \cos (t \Xi)+e_{2} \sin (t \Xi)\right]\right), \\
& A_{2}(t)=\frac{1}{2} e^{-i t \frac{\lambda^{2}}{2}\left(\frac{1}{\Delta_{2}}+\frac{2}{\Delta_{0}}\right)}\left(-D_{12}(0) e^{i t \frac{\lambda^{2}}{4}\left(\frac{1}{\Delta_{2}}-\frac{2}{\Delta_{0}}\right)}+e^{0.5 i t\left(\Delta_{1}+\frac{\lambda^{2}}{\Delta_{0}}\right)}\left[e_{1} \cos (t \Xi)+e_{2} \sin (t \Xi)\right]\right), \\
& A_{4}(t)=e^{0.5 i t\left(\Delta_{1}-\frac{\lambda^{2}}{2 \Delta_{2}}\right)}\left(e_{11} \cos (t \Xi)+e_{22} \sin (t \Xi)\right), \quad A_{3}(t)=A_{3}(0) e^{i t \mho_{1}} .
\end{aligned}
$$

with $e_{1}=\left(A_{1}(0)+A_{2}(0)\right), e_{2}=-\frac{i\left(\Delta_{1}+\frac{\lambda^{2}}{2 \Delta_{2}}\right)}{2 \Xi} e_{1}, e_{11}=0, e_{22}=-\frac{\lambda}{2 \Xi} e_{1}$, and $\Xi=\frac{1}{2} \sqrt{\Delta_{2}^{2}+\frac{\lambda^{4}}{4 \Delta_{2}^{2}}+\frac{\Delta_{1} \lambda^{2}}{\Delta_{2}}+2 \lambda^{2}}$. Given the wave functions in the different regimes, we are having the way to discuss entanglement.

\section{Quantum Entropy}

The physical essence of entanglement consists in the existence of quantum correlations between the individual parts of a composite system that have interacted once in the past but are no longer interacting. Formally, these correlations are caused by combination of the superposition principle in quantum mechanics with the tensor product structure of the space of states [98]. Quantum mechanically, the von Neumann entropy for a quantum system is defined as

$$
S=-\operatorname{Tr} \rho \ln \rho,
$$

where $\rho$ is the density operator for the given quantum system and we have set Boltzmanns constant equal to 1 . Quantum entropies are generally difficult to compute because they involve the diagonalization of large (and, in many cases, infinite dimensional) density matrices. If $\rho$ describes a pure state, then $S=0$, and if $\rho$ describes a mixed state, then $S \neq 0$. Let $S_{A}$ and $S_{F}$ denote the entropies of two interacting systems (i.e A and F) and let $\mathrm{S}$ denotes the entropy of the composite system. Araki and Lieb [99] showed that these entropies satisfy the triangle inequalities

$$
\left|S_{A}-S_{F}\right| \leq S \leq S_{A}+S_{F} .
$$

The entropies of the atom and the field, when treated as a separate system, are defined through the corresponding reduced density operators by

$$
S_{A(F)}=-\operatorname{Tr}_{F(A)}\left(\rho_{A(F)} \ln \rho_{A(F)}\right) .
$$


Here we use the quantum field entropy as a measure of the degree of entanglement between the field and the atoms of the system under consideration. We assume that the system starts from a pure state. Consequently, its entropy $S$ vanishes (i.e. $S=0$ ). Therefore, inequality (32) implies that $S_{A}=S_{F}$. Since the trace is invariant under a similarity transformation, we can go to bases in which the atomic density matrix is diagonal and write Eq.(33) as.

$$
D E M(t)=S_{A}=S_{F}=-\sum_{j=1}^{\text {levels }} \varrho_{j}^{\text {number }} \ln \varrho_{j} .
$$

The $\varrho_{j}$ are the eigenvalues of the atomic density matrix $\rho_{A}(t)$. The bipartite quantum states $\rho_{A F}^{(12)}(t)$ follows $\rho_{A F}^{(12)}(t)=$ $\left|\Psi_{A F}(t)\right\rangle\left\langle\Psi_{A F}(t)\right|$. Thus, the reduced atomic density operator $\rho_{A}^{(12)}(t)=\operatorname{Tr}_{F} \rho_{A F}^{(12)}(t)$ is as follows:-

$$
\rho_{A}^{(12)}(t)=\sum_{i, j k, l=e, g} \rho_{i j, k l}(t)|i j><k l|
$$

Therefore, the eigenvalues $\varrho_{j}(j=1,2,3,4)$ are given by

$$
\begin{gathered}
\varrho_{+}=-\frac{\Upsilon_{1}}{4}+\frac{1}{2}\left(\sqrt{\left\{\alpha+2 y^{(n)}\right\}} \pm \sqrt{\left.\left(\alpha+2 y^{(n)}\right)-4\left(\left(\alpha+y^{(n)}\right)+\frac{\beta}{2 \sqrt{\left\{\alpha+2 y^{(n)}\right\}}}\right)\right)}\right. \\
\varrho_{-}=-\frac{\Upsilon_{1}}{4}+\frac{1}{2}\left(-\sqrt{\left\{\alpha+2 y^{(n)}\right\}} \pm \sqrt{\left.\left(\alpha+2 y^{(n)}\right)-4\left(\left(\alpha+y^{(n)}\right)-\frac{\beta}{2 \sqrt{\left\{\alpha+2 y^{(n)}\right\}}}\right)\right)}\right.
\end{gathered}
$$

where $y$ is a real root of the cubic equation.

$$
y^{3}+\left(\frac{5}{2}\right) \alpha y^{2}+\left(2 \alpha^{2}-\gamma\right) y+\left(\frac{\alpha^{3}}{2}-\frac{\alpha \gamma}{2}-\frac{\beta^{2}}{8}\right)=0
$$

with

$$
\left(\begin{array}{c}
\alpha \\
\beta \\
\gamma
\end{array}\right)=\left(\begin{array}{c}
-\frac{3}{8} \Upsilon_{1}^{2}+\Upsilon_{2} \\
\frac{1}{8} \Upsilon_{1}^{3}-\frac{1}{2} \Upsilon_{1} \Upsilon_{2}+\Upsilon_{3} \\
-\frac{3}{256} \Upsilon_{1}^{4}+\frac{1}{16} \Upsilon_{2} \Upsilon_{1}^{2}-\frac{1}{4} \Upsilon_{1} \Upsilon_{3}+\Upsilon_{4}
\end{array}\right)
$$

while $\Upsilon_{1}, \Upsilon_{2}, \Upsilon_{3}$, and $\Upsilon_{4}$ are respectively the coefficients of the algebraic equation $\left|\rho_{A}(t)-\varrho_{j}\right|=0$. Furthermore, the atomic density operators of the $i^{\text {th }}$-atom can be obtained by tracing over the second atom $\rho_{a}^{(i)}(t)=t r_{\text {second atom }} \rho_{A}(t)$. Where $\varrho_{j}$ are the eigenvalues of the reduced density matrix $\rho_{A}(t)$ for one of the two atoms:

$$
\rho_{a}(t)=\left(\begin{array}{cc}
\rho_{e e} & \rho_{e g} \\
\rho_{g e} & \rho_{g g}
\end{array}\right)
$$

Therefore, the eigenvalues $\varrho_{j}(j=1,2)$ are given by

$$
\varrho_{j}=\frac{1+(-)^{j} \sqrt{1-4\left(\rho_{e e} \rho_{g g}-\rho_{g e} \rho_{e g}\right)}}{2} .
$$

Then, the atomic density matrix for the two atoms, in the basis of $|e e\rangle,|e g\rangle,|g e\rangle,|g g\rangle$, is

$$
\rho_{A}(t)=\left(\begin{array}{cccc}
0 & 0 & 0 & 0 \\
0 & \left|B_{1}(t)\right|^{2} & B_{1}(t) B_{2}^{*}(t) & B_{1}(t) B_{3}^{*}(t) \\
0 & B_{2}(t) B_{1}^{*}(t) & \left|B_{2}(t)\right|^{2} & B_{2}(t) B_{3}^{*}(t) \\
0 & B_{3}(t) B_{1}^{*}(t) & \left.B_{3}(t) B_{2}^{*}(t)\right) & \left|B_{3}(t)\right|^{2}+\sum_{j=4}^{6}\left|B_{j}(t)\right|^{2}
\end{array}\right) .
$$

Then, the atomic density matrix for $1^{\text {st }}$-atom, in the basis of $|e\rangle_{1},|g\rangle_{1}$, is

$$
\rho_{a}^{(1)}(t)=\left(\begin{array}{cc}
\left|B_{1}(t)\right|^{2}+\left|B_{4}(t)\right|^{2} & B_{1}(t) B_{3}^{*}(t) \\
B_{3}(t) B_{1}^{*}(t) & \sum_{j=2}^{3}\left|B_{j}(t)\right|^{2}+\sum_{j=5}^{6}\left|B_{j}(t)\right|^{2}
\end{array}\right) .
$$


Then, the atomic density matrix for $2^{\text {nd }}$-atom, in the basis of $|e\rangle_{2},|g\rangle_{2}$, is

$$
\rho_{a}^{(2)}(t)=\left(\begin{array}{cc}
\left|B_{2}(t)\right|^{2} & B_{3}(t) B_{4}^{*}(t) \\
B_{4}(t) B_{3}^{*}(t) & \left|B_{1}(t)\right|^{2}+\sum_{j=3}^{6}\left|B_{j}(t)\right|^{2}
\end{array}\right) .
$$

Having obtained these atomic density matrices, the properties of the DEMs due to the influences of various inputs of the fields and the atoms can be investigated in accordance with controlling conditions in each dispersive regime, using Eq.(34), and Eqs.(35, 35, 39).

\section{Numerical Results and Discussion}

In this section, we use the obtained analytical results in the previous sections to investigate the evolution of the atomic von Neumann entropies: the atom-atom entanglement, $1^{\text {st }}$-atom entanglement, and $2^{\text {nd }}$-atom entanglement for the system under consideration. We use the scaled time $\tau=\lambda t$. In the numerical computations, the atomic frequency will be taken as $\Omega=\omega_{0} \sec (2 \eta), 0 \leq \eta<\frac{\pi}{4} ; \eta$ is an arbitrary parameter: $\Delta_{0}=\omega_{0}(\sec (2 \eta)-1)$. We consider only the case of the absence of the external classical field. Finally, we assume that all the inputs for the parameters of the fields and the atoms are positive real numbers.

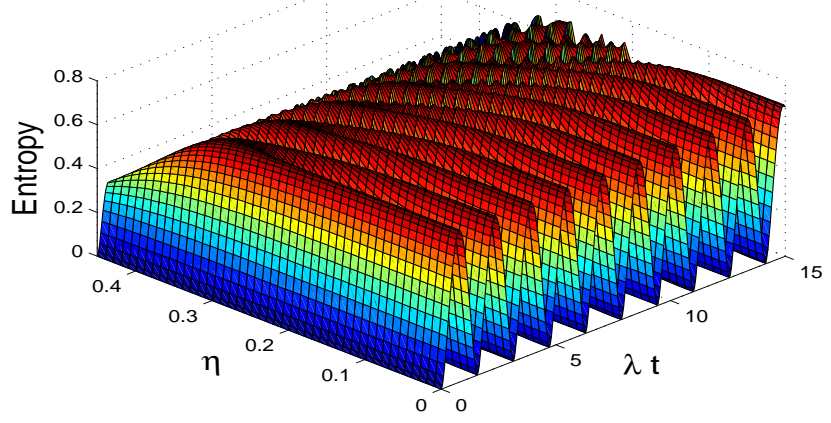

Figure 2: Evolution of the DEM as a function of the scaled time $\lambda t$ for the Hamiltonian in Eq.(1), while $\eta=$ linespace $(0.0: 0.01: 0.45), \nu=0$, and $\omega_{0}=10 \lambda$ with $\left(\theta, \zeta_{j}\right)=\mathbf{0}$.

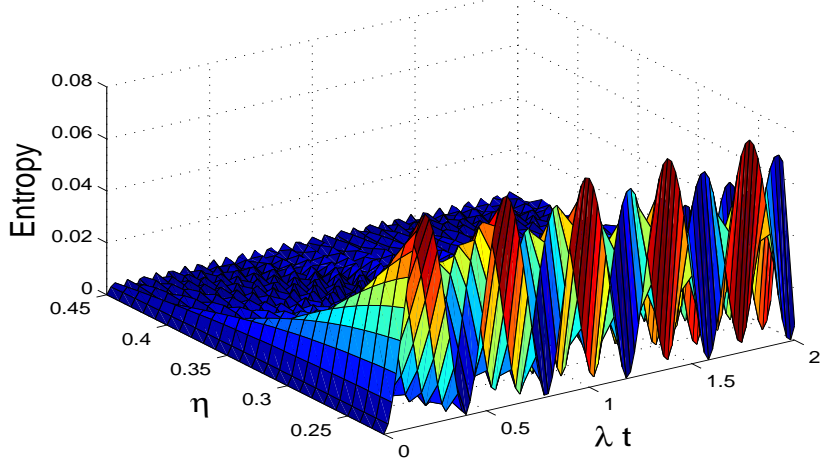

Figure 3: Evolution of the DEM as a function of the scaled time $\lambda t$ for the Hamiltonian in Eq.(1), while $\eta=$ linespace $(0.22: 0.01: 0.45), \nu=0$, and $\omega_{0}=150 \lambda$ with $\left(\theta, \zeta_{j}\right)=\mathbf{0}$.

\subsection{Two Remote (Isolated) Cavities}

To investigate the effect of the localized detuning (i.e., $\Delta_{0}$ ) on the DEM function, we plot the atomic entropies functions against the scaled time and various values of the localized detuning. It is expected that the various values of the detuning provide various features to DEMs. To realize the action of the detuning, we introduce the investigations in accordance with two completely different categories, the first is expressed as $\Delta_{0}<\lambda$, while the opposite $i . e$., $\Delta_{0}>\lambda$ is for the second category. Firstly, when the detuning is of a lower value compared with that for the atom-field coupling, the atomic entropy function exhibits periodical and regular oscillations through the time evolution. Increasing its values leads the maximum values to experience a slight decrease and brings the odd numbered valleys to upward (see Fig.(2)). Secondly, when the detuning is of a larger value compared with that for the atom-field coupling, we observe that its growth causes $(i)$ the maximum values to be brought downward $(i i)$ the function is to oscillate between zero and the maxima in a fast manner with fluctuations: described by almost periodical fluctuations (see Fig.(3)). The increase of the detuning gives rise to the features become more clearer. Finally, we can say that, increasing the detuning reduces the degree of entanglement. Such a reduction occurs faster when the detuning values exceedingly exceed that for the atom-field coupling (compare Fig.(2) with Fig.(3)). Despite that the considered system provides new information different from those in the case of a one-cavity, it is noted that the $2^{\text {nd }}$-atom entanglement is of a zero value through the time evolution. It is interesting to show the amplitudes of the excitations of the 
delocalized bosonic and atomic modes in the mathematical relations framework. In absence of both the action of the optical fiber cable and the detuning, they are formed as follows:-

$$
\left(\begin{array}{c}
A_{1}(t) \\
A_{2}(t) \\
A_{4}(t) \\
A_{5}(t) \\
A_{6}(t)
\end{array}\right)=\left(\begin{array}{c}
A_{1}(0) \cos (\lambda t) \\
A_{2}(0) \cos (\lambda t) \\
-\frac{1}{2} S_{12}(0) \sin (\lambda t) \\
-\frac{1}{2} S_{12}(0) \sin (\lambda t) \\
-\frac{1}{\sqrt{2}} D_{12}(0) \sin (\lambda t)
\end{array}\right) .
$$

Obviously, the excitations of both the fiber mode and the $2^{\text {nd }}$ mode field are zeroes. Also, the above relations show that the $1^{\text {st }}$ mode field and the $1^{\text {st }}$ atom oscillate at the same rate $\lambda$ and with unique fixed amplitude 1 . While, if the optical fiber cable coupling strength is zero and the detuning takes different (i.e., small and large) quantities, the excitations of the delocalized bosonic modes are formed as:-

$$
\begin{aligned}
& A_{o}(t)=-e^{i t \frac{\Delta_{0}}{2}} \frac{\lambda S_{12}(0)}{2 \sqrt{\left(\frac{\Delta_{0}}{2}\right)^{2}+\lambda^{2}}} \sin \left(t \sqrt{\left(\frac{\Delta_{0}}{2}\right)^{2}+\lambda^{2}}\right), o=4,5 \\
& A_{6}(t)=-e^{i t \frac{\Delta_{0}}{2}} \frac{(\lambda / \sqrt{2}) D_{12}(0)}{\sqrt{\left(\frac{\Delta_{0}}{2}\right)^{2}+\lambda^{2}}} \sin \left(t \sqrt{\left(\frac{\Delta_{0}}{2}\right)^{2}+\lambda^{2}}\right),
\end{aligned}
$$

while, we content to display the eigenvalues (roots) corresponding to $A_{1,2}(t)$.

$$
\begin{aligned}
\theta_{j}^{[3]} & =\left(\frac{1}{3} \cos ^{-1}\left[\frac{-9 \lambda^{2} \Delta_{0}-2 \Delta_{0}^{3}}{2\left(\Delta_{0}^{2}+3 \lambda^{2}\right)^{\frac{3}{2}}}\right]+(j-1) \frac{2 \pi}{3}\right), \\
\tau_{j}^{[3]} & =2\left(\frac{\Delta_{0}}{3}\right)+\frac{2}{3}\left(\sqrt{\Delta_{0}^{2}+3 \lambda^{2}}\right) \cos \left(\theta_{j}^{[3]}\right), j=1,2,3 .
\end{aligned}
$$

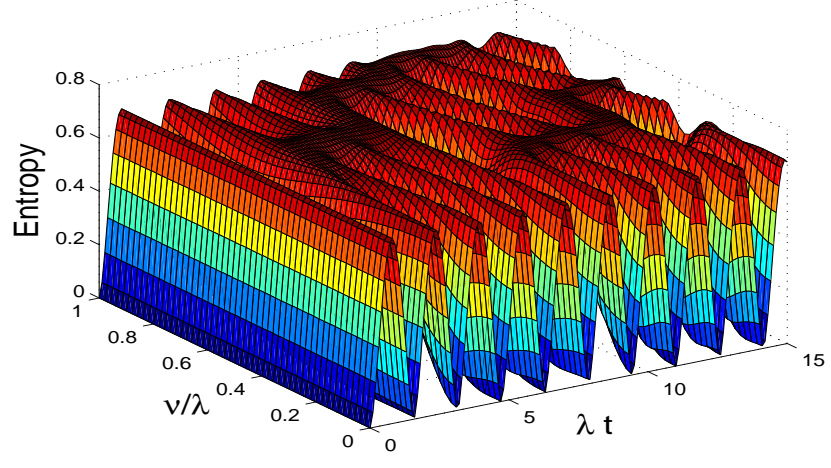

Figure 4: Evolution of the DEM as a function of the scaled time $\lambda t$ for the Hamiltonian in Eq.(1), while $\nu=$ $\lambda$ linespace $(0: 0.02: 1), \eta=0$, and $\omega_{0}=10 \lambda$ with $\left(\theta, \zeta_{j}\right)=\mathbf{0}$.

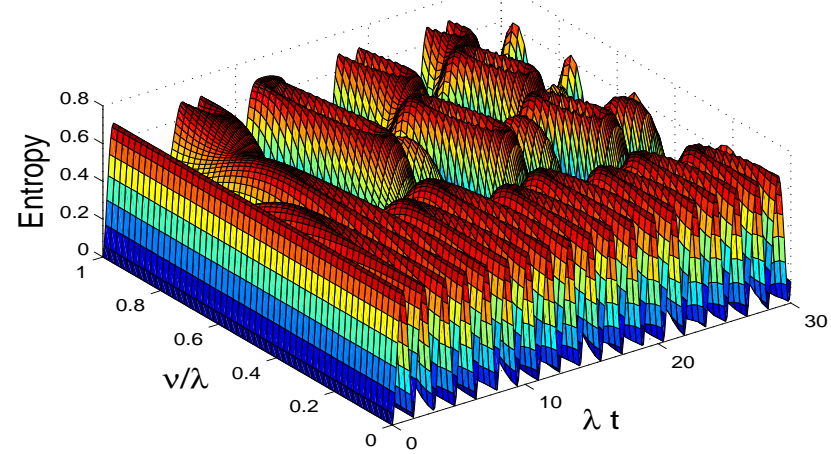

Figure 5: Evolution of the DEM as a function of the scaled time $\lambda t$ for $1^{\text {st }}$-atom of the Hamiltonian in Eq.(1), while $\nu=\lambda$ linespace $(0: 0.02: 1), \eta=0$, and $\omega_{0}=10 \lambda$ with $\left(\theta, \zeta_{j}\right)=\mathbf{0}$.

The immediately stated analytical relations show that the $2^{\text {nd }}$ mode field remains not excited although considering the detuning action. The excitations of the $1^{\text {st }}$ mode field is in continuous reduction as a direct result for the increase of the detuning (i.e., with a huge detuning, $\left.B_{4}(t) \approx 0\right)$. Thus, the fast demolition is for the atom-atom DEM.

\subsection{Two Connected Cavities}

Now, we are going to discuss the behavior of the atomic entropies of the considered system when the strength of the optical fiber cable is of a nonzero value (i.e., $\nu \neq 0)$. The discussion covers three different entropies: namely atom-atom entanglement, $1^{\text {st }}$-atom entanglement, and $2^{\text {nd }}$-atom entanglement through employment of the same inputs in each 
evolution. The aim of such an employment is to realize the behavior of transferring the information amongst the subsystems. Now, we are in a position to realize the action of the existence of the optical fiber cable as a linker between two distant cavities reliance on the case of the absence of both of them.

\subsubsection{Effect of the Optical Fiber Cable Coupling Strength in Absence of the Detuning}

\subsubsection{Two Atoms Entanglement}

Previously, we have showed that the atom-atom DEM function oscillates between zero and its maximum values in a periodic and a regular manner in absence of both the optical fiber cable and the detuning. To show the influence of the strength of the optical fiber cable coupling on the DEMs, we plot the atomic entropies against the scaled time and various values of the optical fiber cable coupling strength. In detail, we work according to two completely different categories $(i) \sqrt{2} \nu<<\lambda,($ ii $) \sqrt{2} \nu>>\lambda$. Firstly, introducing a value of the strength of the optical fiber cable coupling to the numerical computations (say, $\nu=\lambda$ linespace $(0: 0.01: 1), \eta=0$, and $\left.\omega_{0}=10 \lambda\right)$ causes drastic changes in the behavior of the atomic entropy compared with that for the case of the isolated cavities.

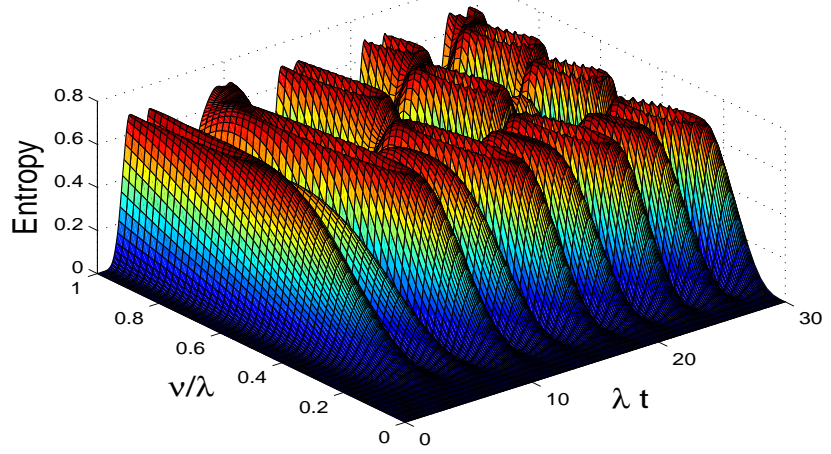

Figure 6: Evolution of the DEM as a function of the scaled time $\lambda t$ for $2^{\text {nd }}$-atom of the Hamiltonian in Eq.(1), while $\nu=\lambda$ linespace $(0: 0.02: 1), \eta=0$, and $\omega_{0}=10 \lambda$ with $\left(\theta, \zeta_{j}\right)=\mathbf{0}$.

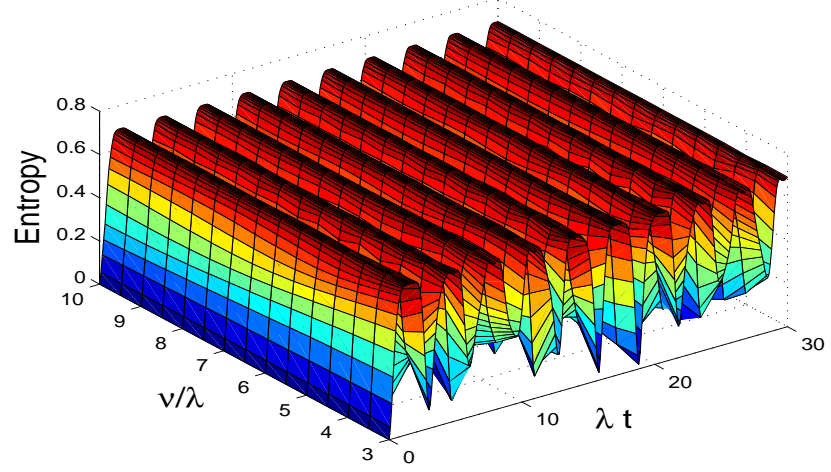

Figure 7: Evolution of the DEM as a function of the scaled time $\lambda t$ for the Hamiltonian in Eq.(1), while $\nu=$ $\lambda$ linespace $(3: 0.5: 10), \omega_{0}=10 \lambda$, and $\eta=0$ with $\left(\theta, \zeta_{j}\right)=\mathbf{0}$.

These features are presented in frequency and amplitude modulations giving rise to successive oscillatory periods. Immediately after the onset of the interaction, the DEM function oscillates between zero and its maxima; as the time goes on, the minima uprise till reach its maximum values for a certain time evolution. The pure states are of a regular occurrence and occur at the midpoint of the oscillatory period (see Fig.(4)). Prolongation and shrinkage of the revivals periods are depend on both the atom-field coupling and the optical fiber cable coupling strength. The small augmentation of the value of the optical fiber cable coupling strength reduces the revival period. Finally, one can ensure that growth the optical fiber cable coupling strength within the depicted interval may control both the number of the oscillations and their regularity. Terminally, introducing a small quantity of the optical fiber cable coupling strength to the numerical computations leads the atom-atom DEM to expose slow and fast oscillations. Meanwhile, it is noticed that the behavior of the atomic entropy when the strength of the optical fiber cable coupling strength exceeds the atom-field coupling is somewhat similar to that for the isolated cavities (say, $\nu=\lambda$ linespace $(3: 0.5: 10)$, $\omega_{0}=10 \lambda$, and $\eta=0$ ) (compare Fig.(4) with Fig.(7)). The strong strength of the optical fiber cable coupling leads to eliminating the fiber mode (see Fig.(7)). Unlike the behavior that for the case of the low strength of the optical fiber cable, the atom-atom DEM is of a monotonic behavior.

\subsubsection{Single Atom Entanglement}

Now, we draw the attention towards the influence of the optical fiber cable coupling strength on the evolution of the $\operatorname{DEM}\left(\rho_{a}^{(i)}(t)\right)$ functions. In the manner of the previous evolutions and employment of the same inputs, the numerical treatments show that the behaviors of the single atoms entanglement are completely different from that for the two atoms entanglement. Firstly; the behavior of the atomic entropy with $(\nu=\lambda \operatorname{linespace}(0: 0.01: 1), \eta=0$, and $\omega_{0}=10 \lambda$ ) shows central peaks with maximum DEM, and intermediate oscillations with far small amplitudes (i.e., each peak is followed by quiescent period). It is obvious from the figures that the first maximum value accelerates 
progressively as the value of the optical fiber cable coupling strength increases (see Fig.(5)). Also, it is observable that the second atom is of disentanglement of the other subsystems when the the lowest values of the strength in the numerical treatments are considered (compare Fig.(5) with Fig.(6)). Secondly; one can observe that the atomic entropy with $\left(\nu=\lambda\right.$ linespace $(3: 0.5: 10), \omega_{0}=10 \lambda$, and $\left.\eta=0\right)$ exhibits oscillatory behavior, it oscillates with a smooth valley followed by spiky one. The smooth valley touches the zero, while, the spiky peaks, their minima increase exponentially and then decrease exponentially through the evolution (see Figs. $(8,9)$ ).

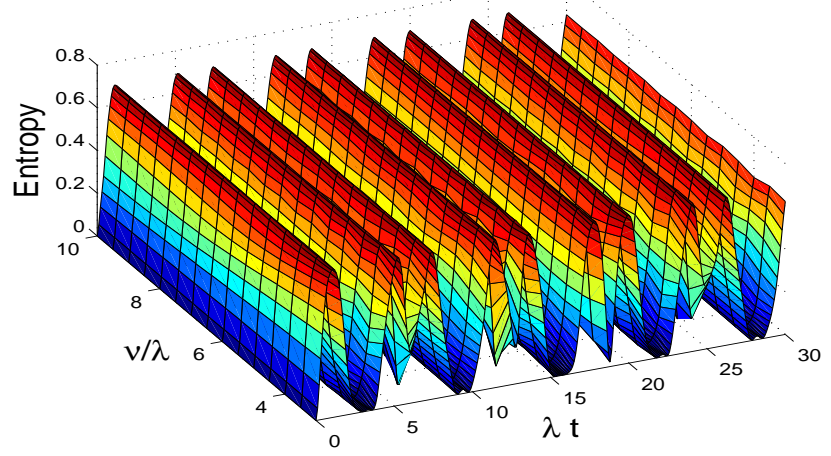

Figure 8: Evolution of the DEM as a function of the scaled time $\lambda t$ for $1^{\text {st }}$-atom of the Hamiltonian in Eq.(1), while $\nu=\lambda$ linespace $(3: .5: 10), \omega_{0}=10 \lambda$, and $\eta=0$ with $\left(\theta, \zeta_{j}\right)=\mathbf{0}$.

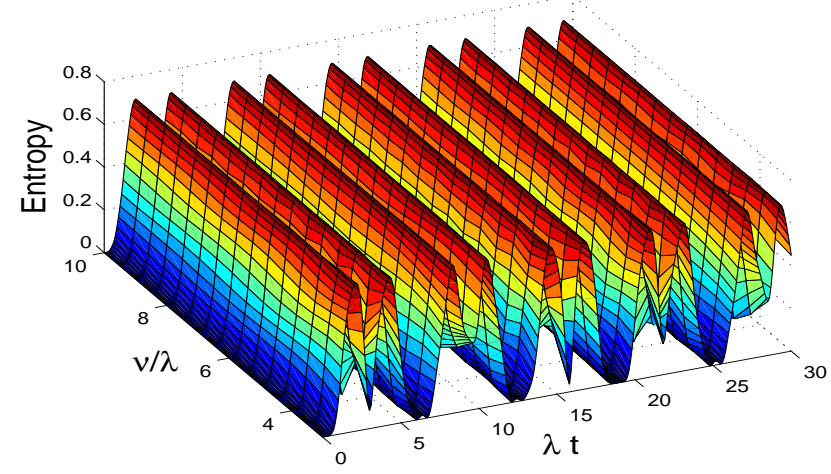

Figure 9: Evolution of the DEM as a function of the scaled time $\lambda t$ for $2^{\text {nd }}$-atom of the Hamiltonian in Eq.(1), while $\nu=\lambda$ linespace $(3: .5: 10), \omega_{0}=10 \lambda$, and $\eta=0$ with $\left(\theta, \zeta_{j}\right)=\mathbf{0}$.

The previous automatically means that the behavior of the atomic entropy is then not monotonic. Given the present situation (i.e., presence of the optical fiber cable coupling strength and absence of the detuning), the amplitudes of the excitations of the delocalized modes can be written as follows:-

$$
\begin{aligned}
& A_{1}(t)=\frac{1}{2}\left[S_{12}(0)-\frac{\lambda^{2}}{\left(2 \nu^{2}+\lambda^{2}\right)} S_{12}(0)+S_{12}(0) \frac{\lambda^{2}}{\left(2 \nu^{2}+\lambda^{2}\right)} \cos \left(t \sqrt{2 \nu^{2}+\lambda^{2}}\right)+D_{12}(0) \cos (t \lambda)\right] \\
& A_{2}(t)=\frac{1}{2}\left[S_{12}(0)-\frac{\lambda^{2}}{\left(2 \nu^{2}+\lambda^{2}\right)} S_{12}(0)+S_{12}(0) \frac{\lambda^{2}}{\left(2 \nu^{2}+\lambda^{2}\right)} \cos \left(t \sqrt{2 \nu^{2}+\lambda^{2}}\right)-D_{12}(0) \cos (t \lambda)\right] \\
& A_{4}(t)=\frac{-\sqrt{2} i \nu \lambda S_{12}(0)}{2\left(2 \nu^{2}+\lambda^{2}\right)}\left(1-\cos \left(t \sqrt{2 \nu^{2}+\lambda^{2}}\right)\right)-\frac{\lambda S_{12}(0)}{2 \sqrt{2 \nu^{2}+\lambda^{2}}} \sin \left(t \sqrt{2 \nu^{2}+\lambda^{2}}\right) \\
& A_{5}(t)=\frac{\sqrt{2} i \nu \lambda S_{12}(0)}{2\left(2 \nu^{2}+\lambda^{2}\right)}\left(1-\cos \left(t \sqrt{2 \nu^{2}+\lambda^{2}}\right)\right)-\frac{\lambda S_{12}(0)}{2 \sqrt{2 \nu^{2}+\lambda^{2}}} \sin \left(t \sqrt{2 \nu^{2}+\lambda^{2}}\right) \\
& A_{6}(t)=-\frac{1}{\sqrt{2}} D_{12}(0) \sin (\lambda t)
\end{aligned}
$$

It is interesting to shed the light on the case of $\sqrt{2} \nu=\lambda$, in it the amplitudes of the five excitations are

$$
\begin{aligned}
& A_{1}(t)=\frac{1}{2}\left[\frac{1}{2} S_{12}(0)+\frac{1}{2} S_{12}(0) \cos (\sqrt{2} \lambda t)+D_{12}(0) \cos (t \lambda)\right] \\
& A_{2}(t)=\frac{1}{2}\left[\frac{1}{2} S_{12}(0)+\frac{1}{2} S_{12}(0) \cos (\sqrt{2} \lambda t)-D_{12}(0) \cos (t \lambda)\right] \\
& A_{4}(t)=\frac{-i S_{12}(0)}{4}(1-\cos (\sqrt{2} \lambda t))-\frac{S_{12}(0)}{2 \sqrt{2}} \sin (\sqrt{2} \lambda t) \\
& A_{5}(t)=\frac{i S_{12}(0)}{4}(1-\cos (\sqrt{2} \lambda t))-\frac{S_{12}(0)}{2 \sqrt{2}} \sin (\sqrt{2} \lambda t) \\
& A_{6}(t)=-\frac{1}{\sqrt{2}} D_{12}(0) \sin (\lambda t) .
\end{aligned}
$$


The stronger the optical fiber cable coupling strength, the amplitudes of the five excitations follow

$$
\begin{aligned}
& A_{1}(t) \approx A_{1}(0) \cos ^{2}\left(\frac{t \lambda}{2}\right)+A_{2}(0) \sin ^{2}\left(\frac{t \lambda}{2}\right) \\
& A_{2}(t) \approx A_{1}(0) \sin ^{2}\left(\frac{t \lambda}{2}\right)+A_{2}(0) \cos ^{2}\left(\frac{t \lambda}{2}\right) \\
& A_{p}(t) \approx 0, A_{6}(t)=-\frac{D_{12}(0)}{\sqrt{2}} \sin (\lambda t), p=4,5
\end{aligned}
$$

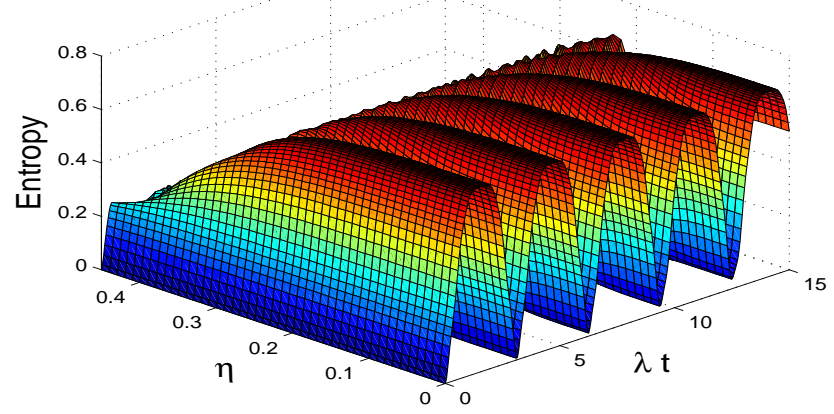

Figure 10: Evolution of the DEM as a function of the scaled time $\lambda t$ for the Hamiltonian in Eq.(1), while $\eta=$ linespace $(0.0: 0.01: 0.45), \nu=10 \lambda$, and $\omega_{0}=10 \lambda$ with $\left(\theta, \zeta_{j}\right)=\mathbf{0}$.

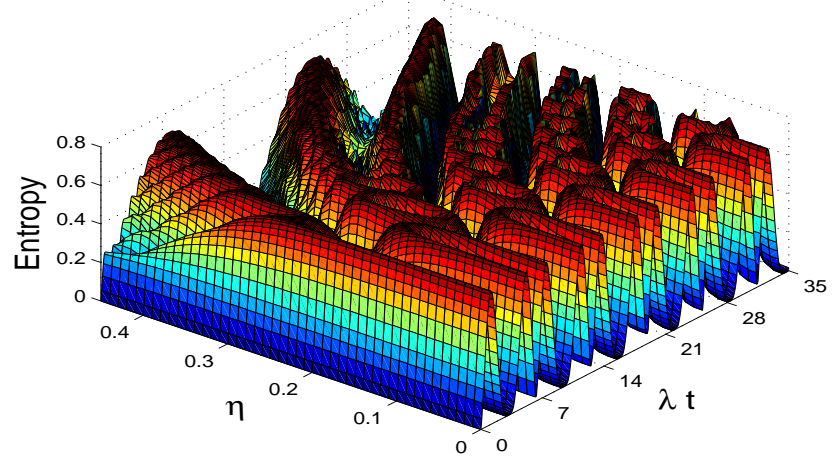

Figure 11: Evolution of the DEM as a function of the scaled time $\lambda t$ for $1^{\text {st }}$-atom of the Hamiltonian in Eq.(1), while $\eta=$ linespace $(0.0: 0.01: 0.45), \nu=10 \lambda$, and $\omega_{0}=10 \lambda$ with $\left(\theta, \zeta_{j}\right)=\mathbf{0}$.

These relations are a direct reference the elimination of the optical fiber mode when a large optical fiber cable strength in account is taken. Also, these relations show that the three atomic entropies under study are of monotonic behaviors. Then, monotonicity of the behaviors of the three atomic entropies under study either occurs when the optical fiber cable is not presented or when it is of huge values on condition the atoms are on resonance with contained fields.

\subsubsection{Effect of the Detuning in Presence of a Large Optical Fiber Cable Coupling Strength}

\subsubsection{Two Atoms Entanglement}

Now, we proceed to know how the atomic entropy behaves as a result of the growth of the detuning whether it exceeds the optical fiber cable coupling strength or not (see Figs.(10, 13)). Aforetime, we have showed that the evolution of the atom-atom entanglement exhibits rapid and regular oscillations fluctuate between zero and maximum values with immutable frequency when the absence of the detuning and the presence of a strong optical fiber cable coupling strength were considered. The addition of the lower values of the detuning $($ say, $\eta=0: 0.01: 0.21$, while $\nu=10 \lambda$, and $\left.\omega_{0}=10 \lambda\right)$ to the numerical computations does not have the ability to add a tangible change into the behavior of the atomic entropy. Continuity of the increase of the detuning on condition that its staying less than the atom-field coupling exposes its (i.e., the atomic entropy) maxima to a slight minimization and its minima to a slight maximization (see Fig.(10)). One can ensure that the behavior of the atom-atom DEM with these poor values compared with that for the atom-field coupling is harmonized with that for the case of the detuning absence. More digression, we have another detuning interval ( say $\eta=0.22: 0.01: 0.45$, while, $\omega_{0}=10 \lambda$, and $\nu=10 \lambda$ ) which is comprehend largeness of the detuning than the atom-field coupling. Firstly, the atom-atom entanglement behavior is greatly affected by such an addendum. The figures show amplitude and frequency modulations. The detuning moves away from the atom-field coupling, the DEM becomes of high frequency and of less extreme values (see Fig.(10)). Now, we terminate by a presentation of another interval, it guarantees exceeding the detuning not only for the atomfield coupling but also for the strength of the optical fiber cable $\left(s a y, \eta=0.22: 0.01: 0.45\right.$, while $\omega_{0}=150 \lambda$, and $\nu=10 \lambda$ ). The atom-atom entanglement is weakened by such an addendum (see Fig.(13)). This reduction results from the fast oscillation of the maximum values of the corresponding atomic entropy and the interference among the revival patterns. The behavior of the fluctuations within the last interval is somewhat losing the regularity. Finally, one can 
emphasize that the detuning is one of the controlling inputs to the amount of the entanglement even in presence of the large optical fiber cable coupling strength.

\subsubsection{Single Atom Entanglement}

The addition of the small values of the detuning (say, $\eta=0: 0.01: 0.21$, while $\omega_{0}=10 \lambda$, and $\nu=10 \lambda$ ) results in modulating the amplitudes and the oscillations. The modulation consists in periodical appearance for oscillatory periods followed by revival one. The end of the oscillatory periods scores zero entanglement. The slight increase in the value of the detuning results in an accelerating the occurrence of the zero entanglement. As long as, the detuning moves towards half of the atom-field coupling, the regularity of the behavior of the modulation of the amplitudes is no longer present as before because of the irregular interference among the revival patterns (see Figs.(11, 12)). Moreover, within an interval that guarantees exceeding the detuning to the atom-field coupling ( $\operatorname{say,~} \eta=0.22: 0.01: 0.45$, while, $\omega_{0}=10 \lambda$, and $\nu=10 \lambda$ ), the numerical treatments show that the evolutions of the single atom evolutions experience dramatic changes: the atomic entropy oscillates in aperiodic manner exhibiting a high frequency serrated valley followed by a high frequency smooth valley (see Figs. $(11,12)$ ). The appearance of serrated valley followed by smooth valley in one of the evolution of the atomic entropies while the opposite in the other is considered a clear indication that the information interchange between the atomic subsystems at specific intervals of time (compare Fig.(11) with Fig.(12)). Finally, the DEMs are greatly affected when the detuning exceeds both the atom-field coupling and the strength of the optical fiber cable coupling: the maxima of the atomic entropy decrease in a rapid manner with increasing the detuning in addition to being the atomic entropy of a high frequency (see Figs. $(14,15)$ ).

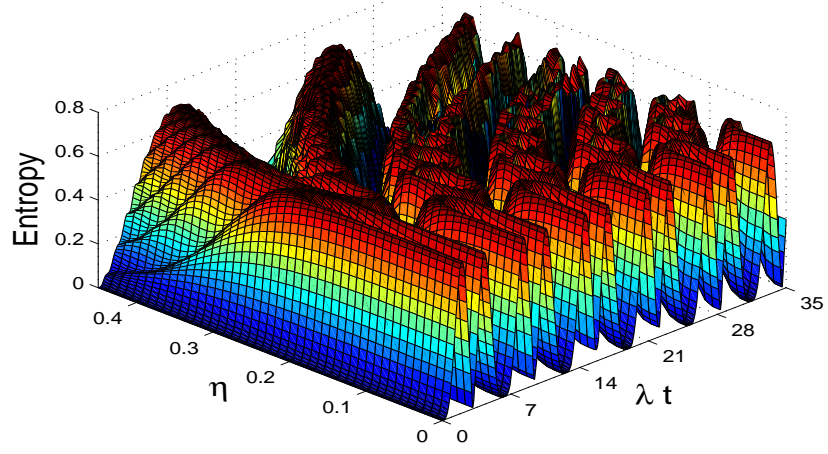

Figure 12: Evolution of the DEM as a function of the scaled time $\lambda t$ for $2^{\text {nd }}$-atom of the Hamiltonian in Eq.(1), while $\eta=$ linespace $(0.0: 0.01: 0.45), \nu=10 \lambda$, and $\omega_{0}=10 \lambda$ with $\left(\theta, \zeta_{j}\right)=\mathbf{0}$.

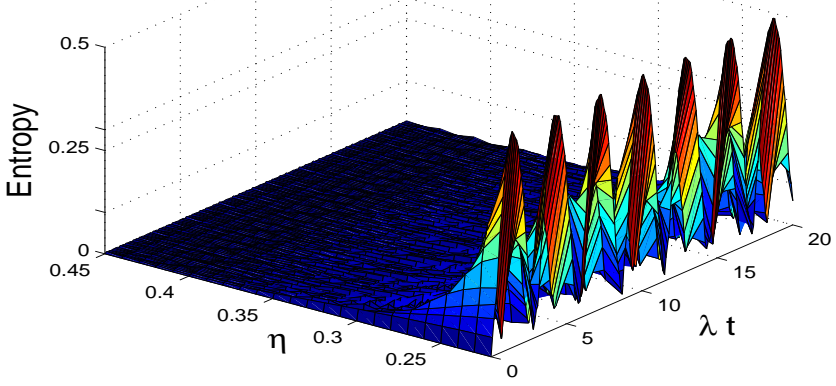

Figure 13: Evolution of the DEM as a function of the scaled time $\lambda t$ for the Hamiltonian in Eq.(1), while $\eta=$ linespace $(0.22: 0.01: 0.45), \nu=10 \lambda$, and $\omega_{0}=150 \lambda$ with $\left(\theta, \zeta_{j}\right)=\mathbf{0}$.

\subsubsection{Effect of the Optical Fiber Cable Coupling Strength in Presence of a Large Detuning}

\subsubsection{Two Atoms Entanglement}

Now, we are proceed towards addressing the atom-atom DEM function of the model under study when the strength of the optical fiber cable coupling is of a value lesser than that for the atom-field coupling while the detuning is of an extremely large value $\left(s a y, \nu=(0: 0.01: 0.5) \lambda\right.$, and $\eta=0.5$, with $\left.\omega_{0}=150 \lambda\right)$. It is known that the DEMs associated with an extreme large value of detuning ( say, the value formed by $\eta=0.5$, with $\omega_{0}=150 \lambda$ ) are of low-level; this can be realized through a closer look to the Fig.(3): therein the atomic entropy function is displayed with $\eta=0.45$, with $\omega_{0}=150 \lambda$. The only marked change observed due to the addition of the sporadic values of the strength of the optical fiber cable coupling consists in an amplitude modulation. The oscillations follow the second order collapses and revivals periods (see Fig.(16)). Now, we are proceed to address the case of exceeding the strength of the optical fiber cable to the atom-field coupling (say, $\nu=(3: 0.125: 10) \lambda$, and $\eta=0.5$, with $\left.\omega_{0}=150 \lambda\right)$. In point of fact, in this case, the behavior of the atomic entropies is somewhat analogous to that with its predecessor. One of what characteristics the present case is being the revival time is far shorter than that for the previous case (compare Fig.(16) with Fig.(19)). Finally, one can ensure that this addition strengthens the DEMs. 


\subsubsection{Single Atom Entanglement}

Considering the large detuning makes the behaviors of the single atoms DEM are somewhat similar to that for atomatom DEM function (compare Fig.(16) with Figs. $(17,18)$ ). The DEMs with the first two evolutions are stronger than that in the last evolution (compare Figs. $(16,17)$ with Fig.(18)). The temporal time evolution directs to that the DEM with the last evolution is of progressively increased maximum values, while, the DEMs with the first two evolutions are of steady maximum values (compare Figs. $(16,17)$ with Fig.(18)). What have been observed from essential differences within the different two intervals of the strength of the optical fiber cable in atom-atom DEM evolution will be for the $1^{\text {st }}$, and $2^{\text {nd }}$ atom DEMs (compare Figs. $(16,17)$ with Figs. $(19,20)$ ) and (compare Fig.(18) with Fig.(21)). The preceding can be realized through a closer look to the last evolution in each case: raising the maximum values to its utmost occurs in a fast manner with the second interval than the first interval (compare Fig.(18) with Fig.(21)). It is obvious that $\theta_{j}^{[l]}$, and $\tau_{j}^{[l]}$ are given by:-

$$
\begin{aligned}
\theta_{j}^{[l]} & =\left(\frac{1}{3} \cos ^{-1}\left[\frac{-9 \lambda^{2} \Delta_{0}-2 \Delta_{0}^{3}+36 \Delta_{0} \nu^{2}}{2\left(\Delta_{0}^{2}+3 \lambda^{2}+6 \nu^{2}\right)^{\frac{3}{2}}}\right]+(j-1) \frac{2 \pi}{3}\right), \\
\tau_{j}^{[1]} & =-\left(\frac{\Delta_{0}-3 \sqrt{2} \nu}{3}\right)+\frac{2}{3}\left(\sqrt{\Delta_{0}^{2}+3 \lambda^{2}+6 \nu^{2}}\right) \cos \left(\theta_{j}^{[1]}\right), j=1,2,3, \\
\tau_{j}^{[2]} & =-\left(\frac{\Delta_{0}+3 \sqrt{2} \nu}{3}\right)+\frac{2}{3}\left(\sqrt{\Delta_{0}^{2}+3 \lambda^{2}+6 \nu^{2}}\right) \cos \left(\theta_{j}^{[2]}\right), j=1,2,3, \\
\tau_{j}^{[3]} & =2\left(\frac{\Delta_{0}}{3}\right)+\frac{2}{3}\left(\sqrt{\Delta_{0}^{2}+3 \lambda^{2}+6 \nu^{2}}\right) \cos \left(\theta_{j}^{[3]}\right), j=1,2,3, .
\end{aligned}
$$

Based on the analytical relation stated now and before in the current text, one can deduce that the rate of the exchanged energy between the atomic subsystems is in a continuous reduction as long as the detuning far exceeds its competition i.e., the optical fiber cable coupling strength and vice versa.

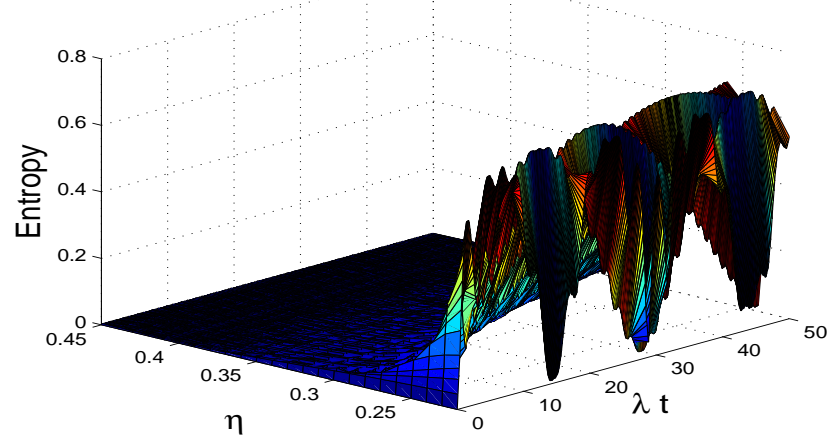

Figure 14: Evolution of the DEM as a function of the scaled time $\lambda t$ for $1^{\text {st }}$-atom of the Hamiltonian in Eq.(1), while $\eta=$ linespace $(0.22: 0.01: 0.45), \nu=10 \lambda$, and $\omega_{0}=150 \lambda$ with $\left(\theta, \zeta_{j}\right)=\mathbf{0}$.

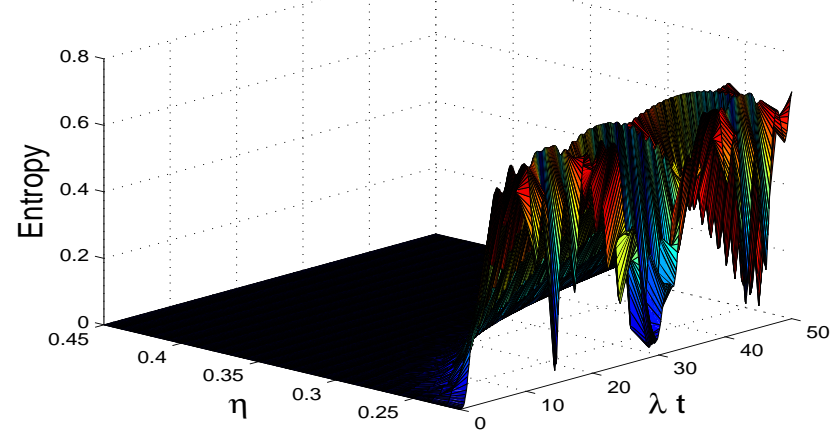

Figure 15: Evolution of the DEM as a function of the scaled time $\lambda t$ for $2^{\text {nd }}$-atom of the Hamiltonian in Eq.(1), while $\eta=$ linespace $(0.22: 0.01: 0.45), \nu=10 \lambda$, and $\omega_{0}=150 \lambda$ with $\left(\theta, \zeta_{j}\right)=\mathbf{0}$.

\subsubsection{Comparable Optical Fiber Cable Coupling Strength and Detuning}

\subsubsection{Two Atoms Entanglement}

To go a step further, we draw the attention to investigate the case in which the detuning is close to the strength of the optical fiber cable coupling (i.e., $\Delta_{0} \approx \sqrt{2} \nu$ ). The previous clearly indicates that the atomic frequency is in near resonant with $\Delta_{1}$. Exceeding the close values to the atom-field coupling causes a reintroduction for oscillating the atomic entropy between zero and its maximum values showing pure states in a periodic manner. Increasing their values propels the atomic entropy to exhibit pure states through the time evolution (see Fig.(22)). Finally, for far large close values, one can observe that the DEM is protected from the irregular demolition during the time evolution. The aforementioned is attributed to being both the valleys and the peaks are of monotonic behavior during the time evolution (see Fig.(22)). 


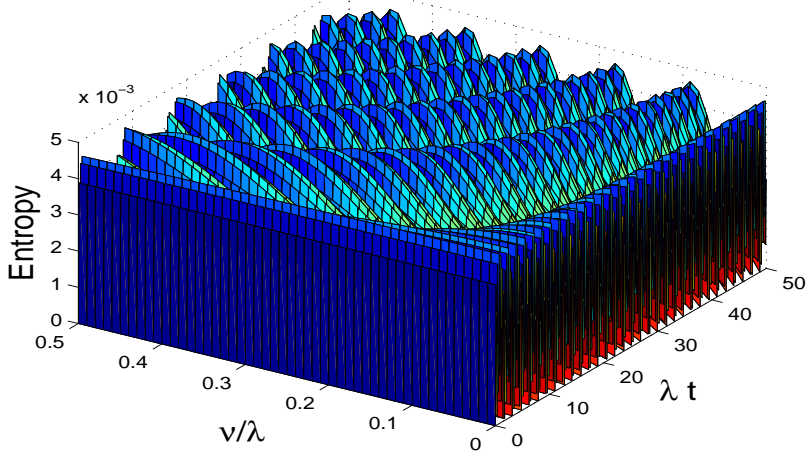

Figure 16: Evolution of the DEM as a function of the scaled time $\lambda t$ for the Hamiltonian in Eq.(1), while $\nu=$ $\lambda$ linespace $(0: 0.01: 0.5), \eta=0.5$, and $\omega_{0}=150 \lambda$ with $\left(\theta, \zeta_{j}\right)=\mathbf{0}$.

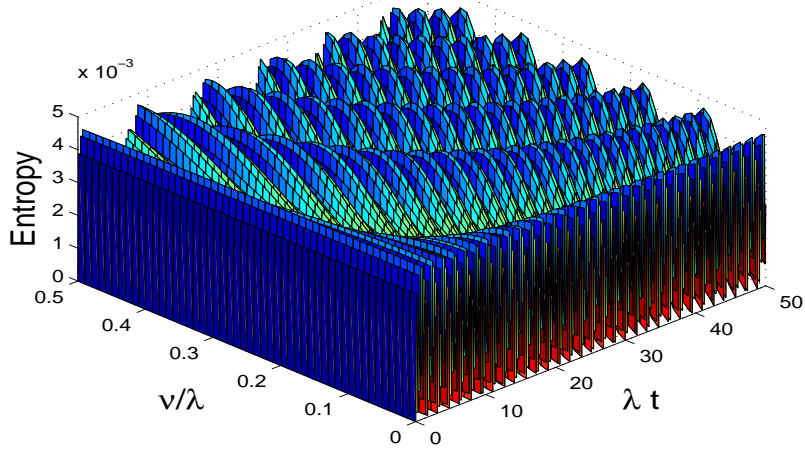

Figure 17: Evolution of the DEM as a function of the scaled time $\lambda t$ for $1^{\text {st }}$-atom of the Hamiltonian in Eq.(1), while $\nu=\lambda$ linespace $(0: 0.01: 0.5), \eta=0.5$, and $\omega_{0}=$ $150 \lambda$ with $\left(\theta, \zeta_{j}\right)=\mathbf{0}$.

The monotonic and regular behavior observed with the larger values of the detuning is attributed to the stability in the behavior of the amplitudes of the excitations of bosonic and fiber modes fields. The reduction of the interatomic energy with the large detuning is attributed to exceeding the amplitudes of the excitation of the fiber mode field to the excitation of both the bosonic modes fields.

\subsubsection{Single Atom Entanglement}

Now, we turn the attention to the study of the single atom DEMs while the values of both the detuning and the strength of the optical fiber cable coupling either comparable to each others or in on resonance. The detuning slightly goes on, the atomic entropy appears eccentricities. Moreover, exceeding the detuning to the half of the atom-field coupling, the atomic entropy exhibits pure states are almost regular. The behavior of the atomic entropy then is a superposition of fast and slow oscillations presented in successive peaks and valleys. Increasing the detuning reduces the revival time and leads the pure states to occur in a fast manner. Furthermore, as long as, the detuning is of high values, the function exhibits valley shape behavior (see Figs.(23, 24)). Again the detuning growth prolongs the revival time. One of what characteristics the behavior of the first atom than the second atom consists in the existence of a phase between them (compare Fig.(23) with Fig.(24)).

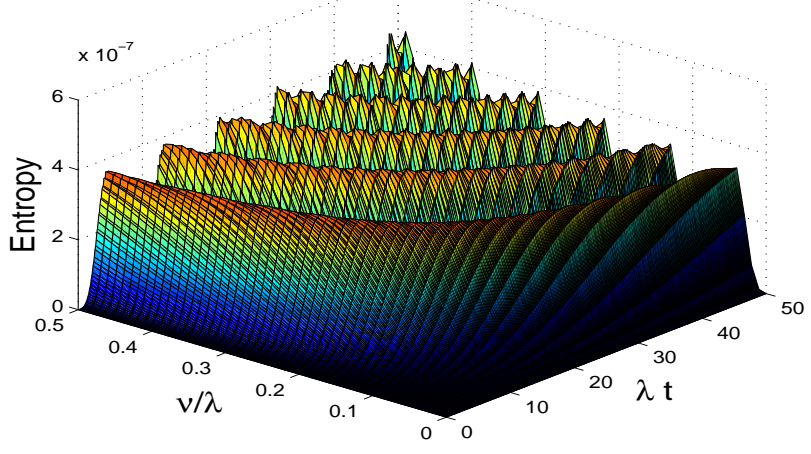

Figure 18: Evolution of the DEM as a function of the scaled time $\lambda t$ for $2^{\text {nd }}$-atom of the Hamiltonian in Eq.(1), while $\nu=\lambda$ linespace $(0: 0.01: 0.5), \eta=0.5$, and $\omega_{0}=$ $150 \lambda$ with $\left(\theta, \zeta_{j}\right)=\mathbf{0}$.

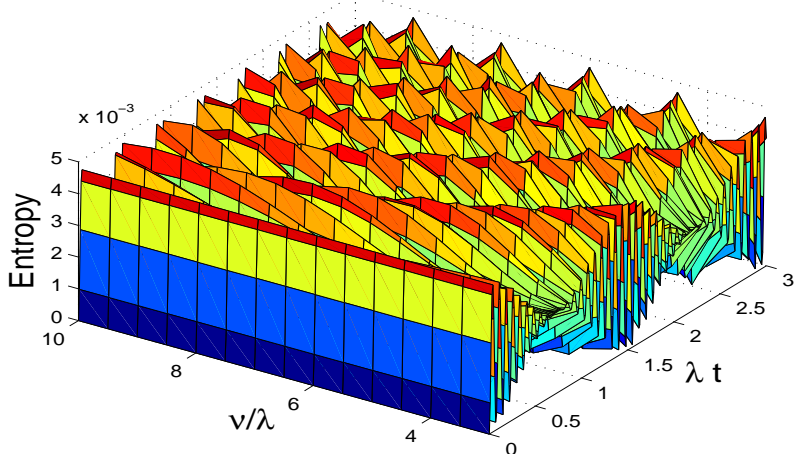

Figure 19: Evolution of the DEM as a function of the scaled time $\lambda t$ for the Hamiltonian in Eq.(1), while $\nu=$ $\lambda$ linespace $(3: 0.125: 10), \eta=0.5$, and $\omega_{0}=150 \lambda$ with $\left(\theta, \zeta_{j}\right)=\mathbf{0}$. 
It is obvious that $\theta_{j}^{[l]}$, and $\tau_{j}^{[l]}$ are given by:-

$$
\begin{aligned}
\theta_{j}^{[l]} & =\left(\frac{1}{3} \cos ^{-1}\left[\frac{-9 \Delta_{0} \lambda^{2}+16 \Delta_{0}^{3}}{2\left(4 \Delta_{0}^{2}+3 \lambda^{2}\right)^{\frac{3}{2}}}\right]+(j-1) \frac{2 \pi}{3}\right), \\
\tau_{j}^{[1]} & =+2\left(\frac{\Delta_{0}}{3}\right)+\frac{2}{3}\left(\sqrt{4 \Delta_{0}^{2}+3 \lambda^{2}}\right) \cos \left(\theta_{j}^{[1]}\right), j=1,2,3, \\
\tau_{j}^{[2]} & =-4\left(\frac{\Delta_{0}}{3}\right)+\frac{2}{3}\left(\sqrt{4 \Delta_{0}^{2}+3 \lambda^{2}}\right) \cos \left(\theta_{j}^{[2]}\right), j=1,2,3, \\
\tau_{j}^{[3]} & =+2\left(\frac{\Delta_{0}}{3}\right)+\frac{2}{3}\left(\sqrt{4 \Delta_{0}^{2}+3 \lambda^{2}}\right) \cos \left(\theta_{j}^{[3]}\right), j=1,2,3 .
\end{aligned}
$$

\subsection{Effect of Coherent Superposition Argument}

It is known and proved that the atomic initial state plays a crucial role in the generation of the entanglement [51]. Based on the analytical and numerical computations, we have observed that the action of the growth of the coherent superposition parameter i.e., $\theta$ (i.e., approaching the $1^{\text {st }}$-atom from its localized ground state) reduces the different DEMs under study (plots are not shown here).

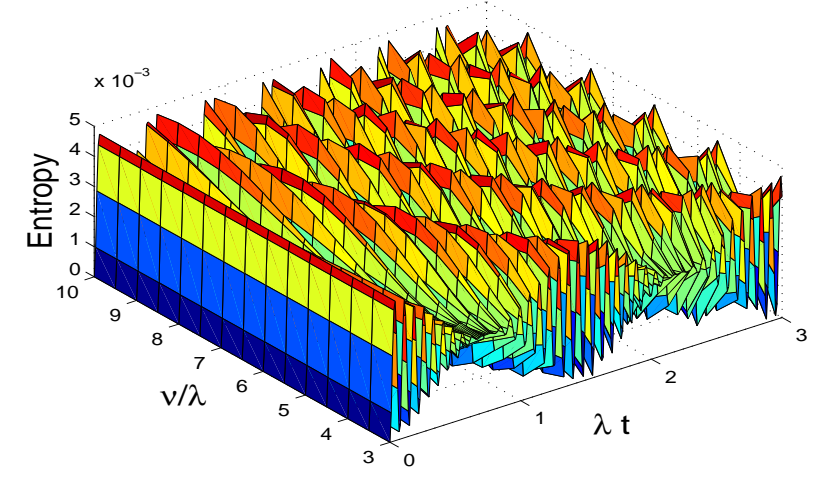

Figure 20: Evolution of the DEM as a function of the scaled time $\lambda t$ for $1^{\text {st }}$-atom of the Hamiltonian in Eq.(1), while $\nu=\lambda$ linespace $(3: 0.125: 10), \eta=0.5$, and $\omega_{0}=150 \lambda$ with $\left(\theta, \zeta_{j}\right)=\mathbf{0}$

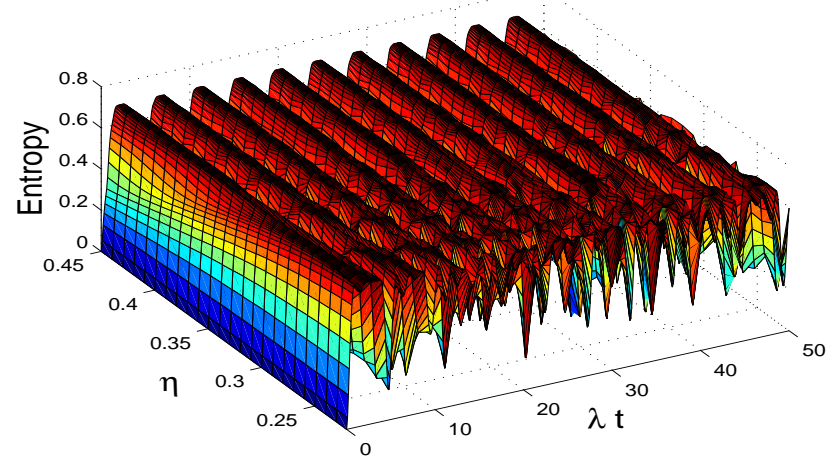

Figure 22: Evolution of the DEM as a function of the scaled time $\lambda t$ for the Hamiltonian in Eq.(1), while $\eta=$ linespace $(0.22: 0.01: 0.45), \nu=\frac{\Delta_{0}}{\sqrt{2}}$, and $\omega_{0}=15 \lambda$ with $\left(\theta, \zeta_{j}\right)=\mathbf{0}$.

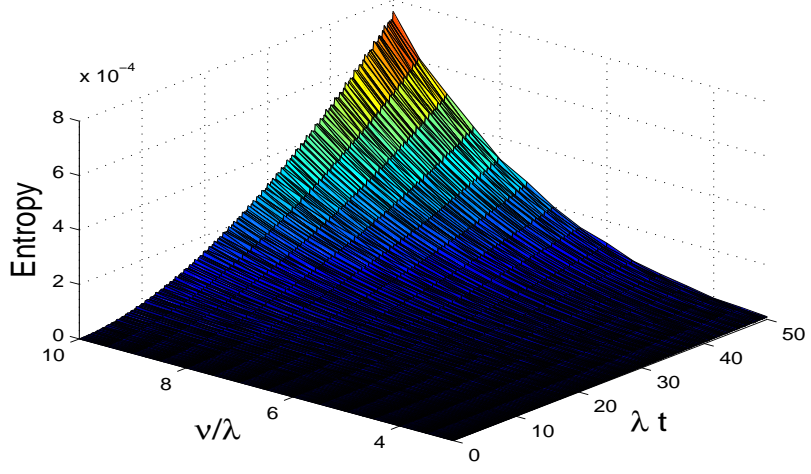

Figure 21: Evolution of the DEM as a function of the scaled time $\lambda t$ for $2^{\text {nd }}$-atom of the Hamiltonian in Eq.(1), while $\nu=\lambda$ linespace $(3: 0.125: 10), \eta=0.5$, and $\omega_{0}=100 \lambda$ with $\left(\theta, \zeta_{j}\right)=\mathbf{0}$.

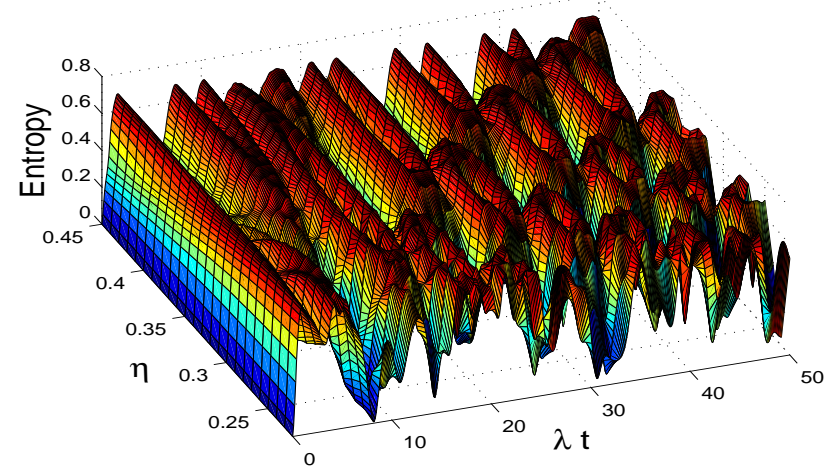

Figure 23: Evolution of the DEM as a function of the scaled time $\lambda t$ for $1^{\text {st }}$-atom of the Hamiltonian in Eq.(1), with $\eta=$ linespace $(0.22: 0.01: 0.45), \nu=\frac{\Delta_{0}}{\sqrt{2}}$, and $\omega_{0}=15 \lambda$. 


\subsection{Time-Average Evolution}

The main target of the current argument is to realize the changes in the DEM functions and its shape when the effective Hamiltonian of time averaged quantum systems is applied. Generally, the time-average technique has its fingerprints clearly on the shape and the strength of the DEM function. It is obvious from the analytical and the numerical results when a large strength of the optical fiber cable is taken into consideration that the impact of the application of such a technique consists in causing a periodic and regular energy transfer between the two atoms without population of the fields. Finally, the time average evolution resembles the exact evolution without the superimposed high frequency components. Reintroduction of both the amplitudes and the relative phase are some of what marked the consideration of the time-average technique (compare Figs.(11, 12) and Figs.(14, 15) with Fig.(25), and Fig.(26) respectively). It should be noted that the previous is the same for the case of the large detuning limit where the only difference between them is limited to the transfer rate (compare Figs.(11, 12) with Figs. $(14,15))$. The large optical fiber cable strength and large detuning effective Hamiltonian of time average quantum system are applied, the strength and the shape of the DEM function follow those for the $2^{\text {nd }}$-atom DEM function when the impact of the time-average techniques were not considered (compare Figs.(17, 18) and Figs.(20, 21) with Fig.(27), and Fig.(28) respectively). Furthermore, the impact of the time average technique with the comparable optical fiber cable coupling strength and detuning case is somewhat different from its counterpart with the large fiber strength and large detuning limits. This is attributed to that the correction of the phase is no longer present as it was in the other two limits. The only observed change is limited to showing a manipulated image with a reintroduction for the regularity in the behavior of the DEM function (compare Figs.(22, 23, 24) with Figs.(29, 30, 31) respectively).

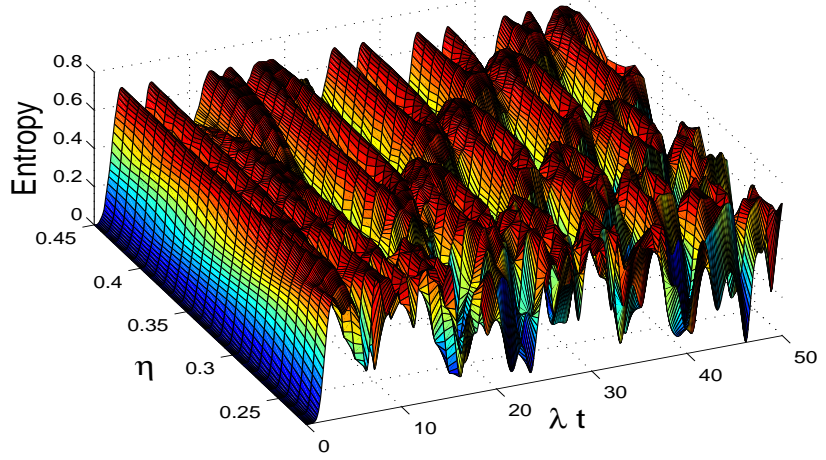

Figure 24: Evolution of the DEM as a function of the scaled time $\lambda t$ for $2^{\text {nd }}$-atom of the Hamiltonian in Eq.(1), while $\eta=\operatorname{linespace}(0.22: 0.01: 0.45), \nu=\frac{\Delta_{0}}{\sqrt{2}}$, and $\omega_{0}=15 \lambda$ with $\left(\theta, \zeta_{j}\right)=\mathbf{0}$.

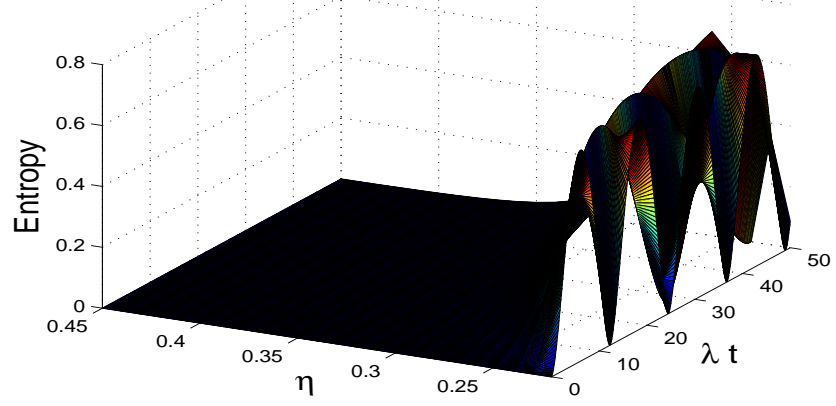

Figure 26: The same as Figs. $(14,15)$, but the Hamiltonian in Eq.(14) is considered.

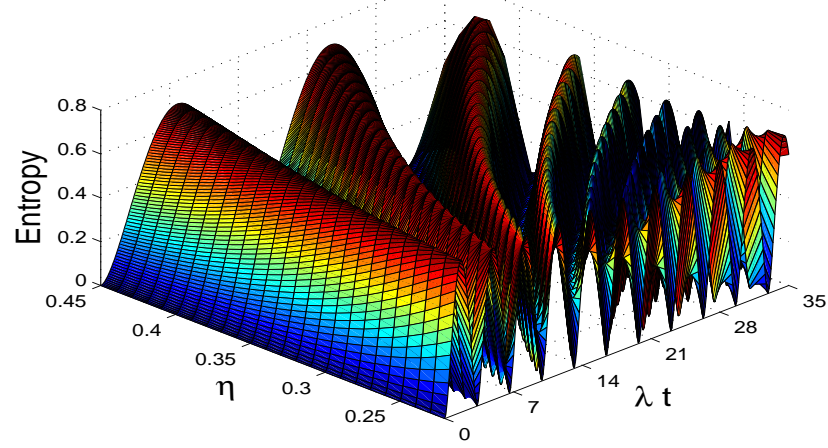

Figure 25: The same as Figs.(11, 12), but the Hamiltonian in Eq.(13) is considered (we cut the interval $0: 0.01: 21$ because of condition negation of the large optical fiber cable strength limit).

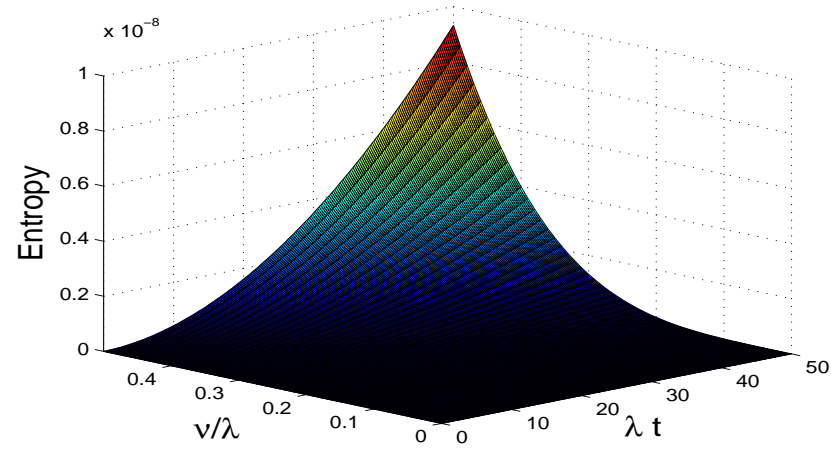

Figure 27: The same as Figs.(17, 18), but the Hamiltonian in Eq.(14) is considered. 


\section{Conclusion}

At this time, we have completed the investigation of the problem of indirectly two coupled cavities; mathematically in the presence of an external classical field and theoretically in its absence case. The effectiveness of both the localized detuning, and the optical fiber cable coupling strength has been completely analyzed. Three different dispersive regime approximations, "their first, is dubbed the large optical fiber cable coupling strength, their second, is dubbed large detuning, and lastly, their third is dubbed the comparable optical fiber cable coupling strength and detunig" and their effectiveness have been investigated. The general conclusions reached and illustrated by numerical results show that:

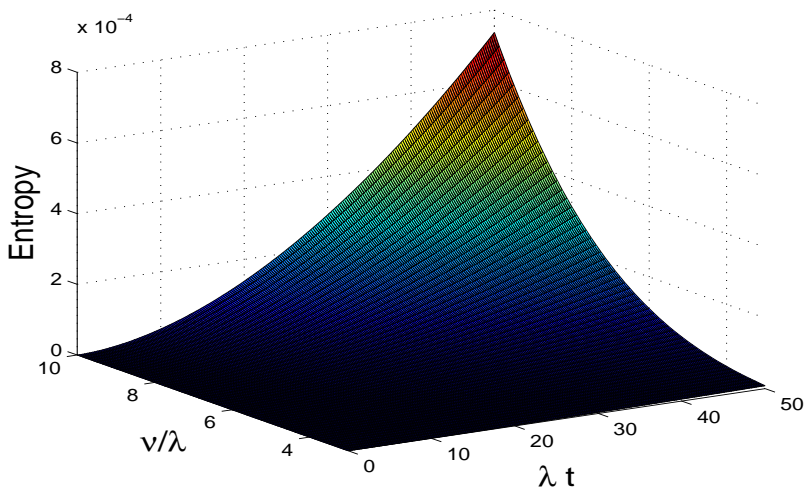

Figure 28: The same as Figs.(20, 21), but the Hamiltonian in Eq.(14) is considered.

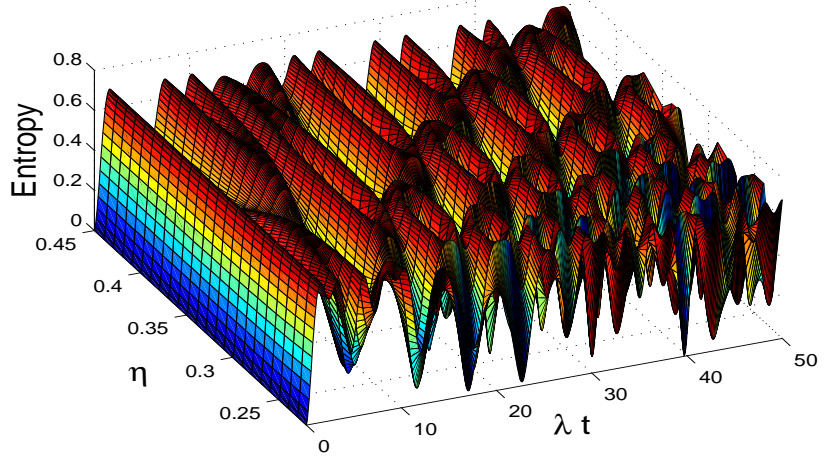

Figure 30: The same as Fig.(23), but the Hamiltonian in Eq.(15) is considered.

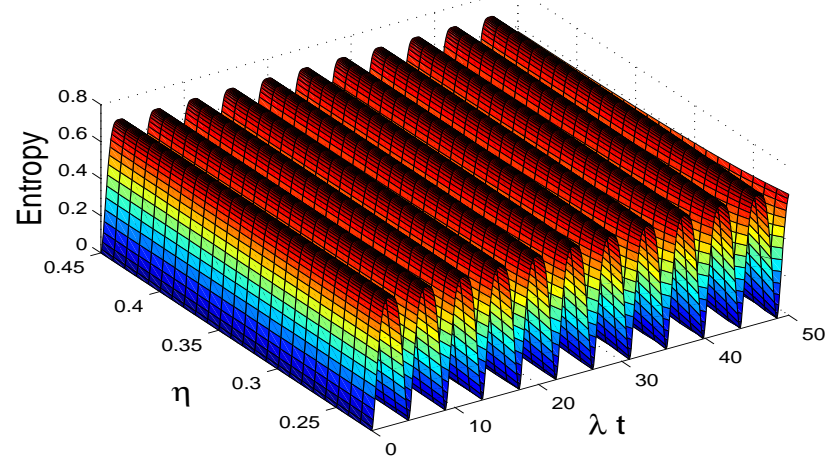

Figure 29: The same as Fig.(22), but the Hamiltonian in Eq.(15) is considered.

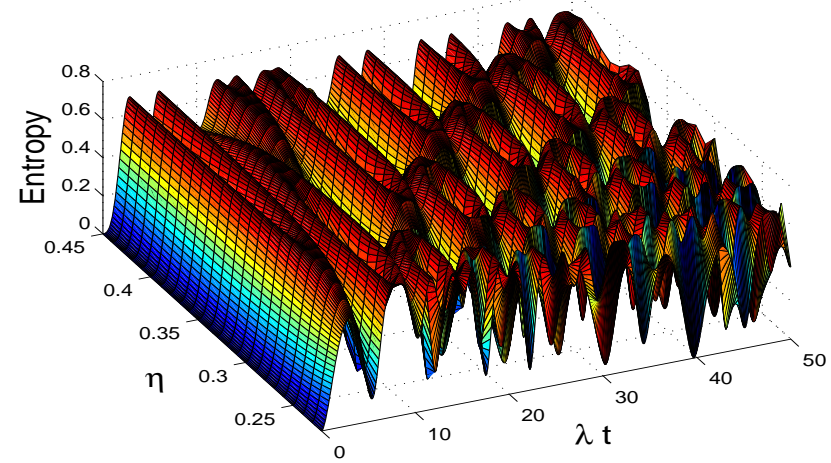

Figure 31: The same as Fig.(24), but the Hamiltonian in Eq.(15) is considered.

- Increment of the detuning provided that its values are small (slightly exceeding) than (to) the atom-field coupling strengthens the DEMs when the strength of the optical fiber cable coupling is inoperative.

- Exceeding the detuning to the atom-field coupling causes a reduction of the DEM" it is presented in the reduction of the maxima of the atomic entropy and the fast interference among the revival patterns" when the strength of the fiber is inoperative.

- Addition of the infinitesimal values of the optical fiber cable coupling strength to while the detuning is inoperative causes an amplitude modulation: it is consist in increasing the minima of the atomic entropy exponentially followed by exponential decrease in a periodic and regular manners, while, continuity of the increase makes a reduction in the revival period.

- Stabilization of the maxima of the atomic entropy is of dependency on the three relevant parameters: the optical fiber cable coupling strength, detuning, and atom-field coupling.

- The stabilization for the maxima of the atomic entropy for specific time instants is clearer seen in the cases of (during)

- Inoperative of both the optical fiber cable coupling strength and the detuning (the evolution of both the atom-atom 
DEM and $1^{\text {st }}$-atom DEM functions).

- Inoperative of detuning and operative of the strength of the optical fiber cable coupling into which exceeds the atom-field coupling (the evolution of the three atomic entropies under study).

- Operative of the detuning on condition that its far smallness than the atom-field coupling and the strength of the optical fiber cable coupling respectively (the evolution of the three atomic entropies under study).

- Operative of both the optical fiber cable coupling strength and the detuning on condition that they are having close values (the evolution of the atom-atom DEM function).

- The optical fiber cable coupling strength is of a larger value compared with that to the detuning, the atomic subsystems give the same DEMs but a phase is observed.

- The optical fiber cable coupling strength is of a smaller value compared with that to the detuning, the atomic subsystems do not give the same DEM.

- Applying the effective Hamiltonian of time averaged quantum systems drives the atomic subsystems to give the same information as long as one of the two effective parameters is much larger than the other.

- Overlapping both $\Delta_{0}$, and $\sqrt{2} \nu$ stabilizes both the strengthes and the associated features of the DEM in the evolution of the atom-atom DEM.

- The exchanged energy rate between the atomic subsystems is controlled by many factors vary in different limits.

- With large optical fiber cable coupling strength and large detuning limits, this controlling factor is $\frac{\lambda^{2}}{4}\left(\frac{1}{\Delta_{1}}+\frac{1}{\Delta_{2}}-\frac{2}{\Delta_{0}}\right)$.

- With the comparable optical fiber cable coupling strength and detuning, this controlling factor is $\frac{\lambda^{2}}{4}\left(\frac{1}{\Delta_{2}}-\frac{2}{\Delta_{0}}\right)$.

\section{Acknowledgment}

The authors wish to thank the referees for their valuable comments which resulted in better readability of the paper.

\section{References}

[1] J. I. Cirac, A. K. Ekert, S. F. Huelga, and C. Macchiavello. Phys. Rev. A, 59:4249, (1999).

[2] M. S. Zubairy, M. Kim, and M. O. Scully . Phys. Rev. A, 68:033820, (2003).

[3] A. Wallraff, D. I. Schuster, A. Blais, L. Frunzio, R. S. Huang, J. Majer, S. Kumar, S. M. Girvin, and R. J. Schoelkopf. Nature (London), 431:162, (2004).

[4] D. I. Schuster, A. A. Houck, J. A. Schreier, A. Wallraff, J. M. Gambetta, A. Blais, L. Frunzio, J. Majer, B. Johnson, M. H. Devoret et al. Nature (London), 445:515, (2007).

[5] A. Vaglica, and G. Vetri . Phys. Rev. A, 75:062120, (2007).

[6] Z. Ficek, and R. Tanas. Phys. Rev. A, 74:024304, (2006).

[7] D. Vitali, S. Gigan, A. Ferreira, H. R. Bohm, P. Tombesi, A. Guerreiro, V. Vedral, A. Zeilinger, and M. Aspelmeyer . Phys. Rev. Lett., 98:030405, (2007).

[8] Q. Zhang, and E. Y. Zhang. Acta Phys. Sin., 51:1684, (2002).

[9] L. Ye, and G. C. Guo. Chin. Phys., 11:996, (2002).

[10] C. H. Bennett, G. Brassard, C. Crepeau, R. Jozsa, A. Peres, and W. K. Wootters. Phys. Rev. Lett., 70:1895, (1993).

[11] N. Metwally, M. Abdelaty, and A.-S. F. Obada. Chaos, Solitons and Fractals, 22:529, (2004).

[12] A. K. Ekert. Phys. Rev. Lett., 67:661, (1991).

[13] C. H. Bennett, and S. J. Wiesner . Phys. Rev. Lett., 69:2881, (1992). 
[14] M. Yang, W. Song, and Z. L. Cao. Phys. Rev. A, 71:034312, (2005).

[15] M. A. Nielsen, and I. L. Chuang. Quantum computation and quantum information, Cambridge, England, (2000).

[16] C. H. Bennett, P. W. Shor, J. A. Smolin, and A. V. Thapliyal . Phys. Rev. Lett., 83:3081, (1999).

[17] S. J. D. Phoenix, and P. L. Knight. Phys. Rev. A, 44:6023, (1991).

[18] S. J. D. Phoenix, and P. L. Knight. Phys. Rev. Lett., 66:2833, (1991).

[19] E.T. Jaynes, and F.W. Cummings. Proc. IEEE, 51:2833, (1963).

[20] A. -S. F. Obada, H. A. Hessian, and M. Hashem. Int. J. Theor. Phys., 48:3643, (2009).

[21] A. -S. F. Obada, M. M. A. Ahmed, F. K. Faramawy, and E. M. Khalil. Chaos, Solitons and Fractals, 28:983, (2006).

[22] M. Abdel-Aty, S. Abdel Khalek, and A. -S. F. Obada . Chaos, Solitons and Fractals, 12:2015, (2001).

[23] M. Sebawe Abdalla, M. Abdel-Aty, and A. -S. F. Obada. International Journal of Theoretical Physics, 44:1649, (2009).

[24] Xi-Wen Hou, Jing-Hua Chen, Ming-Fang Wan, and Zhong-Qi Ma . Optics Communications , 266:727, (2006).

[25] Vladimír Bužek, and Igor Jex. Journal of Modern Optics, 36:1427, (1989).

[26] V. Bužek, H. Moya-Cessa, P. L. Knight, and S. J. D. Phoenix. Physical Review A, 45:8190, (1992).

[27] E. K. Bashkirov, and M. S. Rusakova. Optics Communications , 281:4380, (2008).

[28] Guo-feng Zhang, and Zi-yu Chen. Optics Communications, 275 :274, (2007).

[29] M. Sebawe Abdalla, Mahmoud Abdel-Aty, and A. -S. F. Obada. Physica A , 326:203, (2003).

[30] S. J. Akhtarshenas. International Journal of Theoretical Physics, 45:1005, (2006).

[31] Lei Tan, Zhong-Hua Zhu, and Yu-Qing Zhang. arXiv: 090\%. 3798v2 [quant-ph] and submitted to Phys. Scr., (2010).

[32] A. -S. F. Obada, A. M. Abdel-Hafez, and H. A. Hessian. Acta Physica Slovaca, 52:419, (2002).

[33] A. -S. F. Obada, M. M. A. Ahmed, F. K. Faramawy, and E. M. Khalil. Chinese Journal of Physics, 42:79, (2004).

[34] A. -S. F. Obada, A. M. Abdel-Hafez, and H. A. Hessian. International Journal of Theoretical Physics, 39:1499, (2000).

[35] Z. Ficek. Appl. Math. Inf. Sci., 3:375, (2009).

[36] A. El-Barakaty, M. Darwish, and A. -S. F. Obada. Appl. Math. Inf. Sci., 5:122, (2011).

[37] Li- Hui Sun, Gao-Xiang Li, and Z. Ficek. Appl. Math. Inf. Sci., 4:315, (2010).

[38] M. Sebawe Abdalla, and L. Thabet. Appl. Math. Inf. Sci., 5:570, (2011).

[39] Abdel-Shafy F. Obada, and Mahmoud Abdel-Aty. Eur. Phys. J. D, 27:277, (2003).

[40] R. A. Zait. Physics Letters A, 319:461, (2003).

[41] Qing-Chun Zhou, and Shi Ning Zhu . Optics Communications, 248:437, (2005).

[42] A. -S. F. Obada, A. A. Eied, and G. M. Abd Al-Kader. Int J Theor Phys, 48:380, (2009).

[43] Chunjia Huang, Lijun Tang, Fanzhi Kong, Jiayuan Fang, and Ming Zhou. Physica A, 368:25, (2006).

[44] Mahmoud Abdel-Aty. Laser Physics, 11:871, (2001).

[45] Xiang Liu. Physica A, 286:588, (2000). 
[46] Mihir Ranjan Nath, Surajit Sen, Asoke Kumar Sen, and Gautam Gangopadhyay. Pramana Journal Of Physics, 71:77, (2008).

[47] F. C. Lourenco, and A. Vidiella-Barrancoa. Eur. Phys. J. D, 47 :127, (2008).

[48] A.-S. F. Obada, S. A. Hanoura, and A. A. Eied. Eur. Phys. J. D, 66:221, (2012).

[49] A.-S. F. Obada, S. A. Hanoura, and A. A. Eied. Laser Phys., 23:025201, (2013).

[50] A.-S. F. Obada, S. A. Hanoura, and A. A. Eied. Eur. Phys. J. D, 68:18, (2014).

[51] A.-S. F. Obada, S. A. Hanoura, and A. A. Eied. Laser Phys., 24: 055201, (2014).

[52] A. -S. F. Obada, and A. A. Eied. Optics Communications, 282: 2184, (2009).

[53] Yun-Xia Ping, Bo Zhang, Ze Cheng, and Yan-Min Zhang. Physics Letters A, 362:128, (2007).

[54] A.-S. F. Obada, S. A. Hanoura, and A. A. Eied. Laser Phys., 23:055201, (2013).

[55] A.-S. F. Obada, S. A. Hanoura, and A. A. Eied. Optik, 136:602, (2017).

[56] A.-S. F. Obada, S. A. Hanoura, and A. A. Eied. Int. J. Theor. Phys., 51:2665, (2012).

[57] M. Bina, F. Casagrandea, and A. Lulli. Eur. Phys. J. D, 49:257, (2008).

[58] Yan-Hui Wang, Liang Hao, Xiang Zhou, and Gui Lu Long . Optics Communications , 281:4793, (2008).

[59] M. A. A. El-Deberky. International Journal of Physical Sciences, 4:253, (2009).

[60] S. Gong and Y. Niu. Optics and Spectroscopy, 99:270, (2005).

[61] N. H. Abdel-Wahab. J. Phys. B: At. Mol. Opt. Phys., 41:105502(7pp), (2008).

[62] Xin-You Lü, Liu-Gang Si, Meng Wang, Su-Zhen Zhang, and Xiaoxue Yang. J. Phys. B: At. Mol. Opt. Phys., 41:235502, (2008).

[63] F K Nohama, and J A Roversi. J. Phys. B: At. Mol. Opt. Phys., 41:045503, (2008).

[64] Smail Bougouffa, and Zbigniew Ficek. Phys. Rev. A, 88:022317, (2013).

[65] Duong Hai Trieu. Journal of Physics: Conference Series, 187:012032, (2009).

[66] Ke Zhang, and Zhi-Yuan Li. Phys. Rev. A, 81:033843, (2010).

[67] Marco Enríyquez, Claudia Quintana, and Oscar Rosas-Ortiz. Journal of Physics: Conference Series, 512:012022, (2014).

[68] Muhammed Yönac, and J. H. Eberly. Phys. Rev. A, 82:022321, (2010).

[69] Muhammed Yöna, and Joseph H. Eberly. Opt. Lett., 33:270, (2008).

[70] P. Blanco, and D. Mundarain. J. Phys. B: At. Mol. Opt. Phys., 44:105501, (2011).

[71] Hu Yao-Hua, and Wang Jun-Qiang. Chin. Phys. B, 21:014203, (2012).

[72] W. Son, M. S. Kim, Jinhyoung Lee, and D. Ahn. Journal of Modern Optics, 49:1739, (2002).

[73] Hong-Mei Zou, and Mao-Fa Fang. Quantum Inf. Process, 14:2673, (2015).

[74] Qing-Hu Chen, Yuan Yang, Tao Liu, and Ke-Lin Wang. Phys. Rev. A, 82:052306, (2010).

[75] Stanley Chan, M D Reid, and Z Ficek. J. Phys. B: At. Mol. Opt. Phys., 42:065507, (2009).

[76] Isabel Sainz, and Gunnar Björk. Phys. Rev. A, 76:042313, (2007).

[77] Jinhyoung Lee, M. Paternostro, M. S. Kim, and S. Bose. Phys. Rev. Lett., 96:080501, (2006).

[78] Muhammed Yöna, and Joseph H. Eberly. J. Phys. B: At. Mol. Opt. Phys., 39:S621, (2006). 
[79] Bao-Liang Cao, Ying Shi, and Dong-Guang Jiang. Int. J. Theor. Phys., 53:1920, (2014).

[80] S. Bougouffa. Opt. Commun., 283:2989, (2010).

[81] R. Tahira, M. Ikram, S. Bougouffa, and M. S. Zubairy. J. Phys.B: At. Mol. Opt. Phys., 43:035502, (2010).

[82] S. Bougouffa, and A. Hindi. Phys. Scr. T, 143:014006, (2011).

[83] S. Bougouffa. AIP Conf. Proc., 1370:192, (2011).

[84] S. Bougouffa, and Z. Ficek. Phys. Scr. T, 147:014005, (2012).

[85] Daniel F. V. James, and Jonathan Jerke. Can. J. Phys, 85:625, (2007).

[86] C. D. Ogden, E. K. Irish, and M. S. Kim. Phys.Rev.A, 78:063805, (2008).

[87] P. Alsing, D.-S. Guo, and H.J. Carmichael. Physical Review A, 45:5135, (1992).

[88] B.T.H. Varcoe, S. Brattke, B.G. Englert, and H. Walther. Laser Physics, 10:1, (2000).

[89] B.T.H. Varcoe, S. Brattke, M. Weldinger, and H. Walther. Nature, 403:743, (2000).

[90] Q. A. Turchette. Physical Review Letters, 75:4710, (1995).

[91] A. Rauschenbeutel, G. Nogues, S. Osnaghi, P. Brune, J.M. Raimond, and S. Haroche. Physical Review Letters, 83:5166, (1999).

[92] E. M. Khalil. Int J Theor Phys, 52:1122, (2013).

[93] M. Sebawe Abdalla, A-S. F. Obada, E. M. Khalil, and S. I. Ali. Laser Phys, 23:115201, (2013).

[94] M. Sebawe Abdalla, A-S. F. Obada, and E. M. Khalil. Annals of Physics, 326:2486, (2011).

[95] Li-Tuo Shen, Zhen-Biao Yang, Huai-Zhi Wu, Xin-Yu Chen, and Shi-Biao Zheng. J. Opt. Soc. Am. B, 29:2379, (2012).

[96] Peng Jun, Wu Yun-Wen, and Li Xiao-Juan. Chin. Phys. B, 21:060302, (2012).

[97] William Henry Louisell. Quantum statistical properties of radiation. John Wiley, and Sons Canada, (1973).

[98] M. B. Plenio, and V. Verdal. Contemp. Phys., 39:431, (1998).

[99] H. Araki, and E. Lieb. Commun. Math. Phys., 18:160, (1970). 